\title{
Inhibiting DDX3X triggers tumor-intrinsic type I interferon response and enhances anti-tumor immunity
}

Hyeongjwa Choi ${ }^{1}$, Juntae Kwon ${ }^{1}$, Jiafang Sun ${ }^{1}$, Min Soon Cho ${ }^{3}$, Yifan Sun ${ }^{4}$, John L Casey ${ }^{3}$, Jeffrey Toretsky ${ }^{1,2}$, Cecil Han ${ }^{1, \#}$

${ }^{1}$ Department of Oncology, ${ }^{2}$ Departments of Pediatrics, ${ }^{3}$ Department of Microorganism and Immunology, Georgetown University School of Medicine, Washington, D.C. 20007, USA;

${ }^{3}$ Department of Pulmonary Medicine, Division of Internal Medicine, The University of Texas MD Anderson Cancer Center, Houston, TX 77030, USA;

${ }^{4}$ Department of Medical and Molecular Genetics, IU School of Medicine; Indianapolis, IN 46202, USA

${ }^{\#}$ Correspondence: ch1182@georgetown.edu (C.H.) 


\begin{abstract}
Accumulating evidence has shown that cellular double-stranded RNAs (dsRNAs) induce antiviral innate immune responses in human normal and malignant cancer cells. However, it is not fully understood how endogenous 'self' dsRNA homeostasis is regulated in the cell. Here, we show that an RNA-binding protein, DEAD-box RNA helicase 3X (DDX3X), prevents the aberrant accumulation of cellular dsRNAs. Loss of DDX3X induces dsRNA sensor-mediated type I interferon signaling and innate immune response in breast cancer cells due to abnormal cytoplasmic accumulation of dsRNAs. Dual depletion of DDX3X and a dsRNA-editing protein, ADAR1 synergistically activates the cytosolic dsRNA pathway in breast cancer cell. Moreover, inhibiting DDX3X enhances the antitumor activity by increasing tumor intrinsic-type I interferon response, antigen presentation, and tumor-infiltration of cytotoxic $T$ cells as well as dendritic cells in breast tumors, which may lead to the development of breast cancer therapy by targeting DDX3X in combination with immune checkpoint blockade.
\end{abstract}




\section{INTRODUCTION}

Double-stranded RNAs (dsRNAs) within cells are often a result of infected virus replication and considered as a "danger signal" by the host immune system ${ }^{1}$. Upon recognition of these "non-self" dsRNAs, the host cytoplasmic dsRNA sensors trigger type I Interferon (IFN) production and induce antiviral responses via upregulation of IFN-stimulated genes (ISGs), that eventually leads to cellular apoptosis or growth inhibition ${ }^{2,3}$. These dsRNA sensors include Tolllike receptor family proteins (TLR3, TLR7), retinoic acid inducible gene I (RIG-I), melanoma differentiation associated gene 5 (MDA5), and protein kinase $R(P K R)^{1,4,5}$. In addition, another dsRNA binding protein, 2'-5'-oligoadenylate synthetase 1 (OAS1) directly acts on viral RNA by inducing its degradation ${ }^{1}$.

Advanced transcriptomics studies have shown that metazoan cells express various types of endogenous "self" dsRNAs such as endogenous retroviral elements (ERVs), repetitive RNA elements, mitochondrial dsRNAs, mRNAs with inverted Alu-containing 3' UTRs, and structural dsRNAs with long dsRNA stem ${ }^{6-11}$. Current evidence has revealed that abnormal accumulation of the endogenous dsRNAs in mammalian cells could trigger antiviral innate immune response through activation of dsRNA sensing pathways, such as MDA5 or PKR, and that this response could cause chronic inflammation and related human diseases ${ }^{3,12,13}$. In particular, intracellular dsRNA accumulation in tumors stimulates induction of cancer-derived type I IFN, which enhances anti-tumor immunity ${ }^{14-18}$. Of note, ERVs constitute more than $8 \%$ of the human genome and their bi-directional transcription has been shown to increase the formation of dsRNAs ${ }^{19-21}$. DNA methylation silences most ERVs in normal somatic cells, but some cancers exhibit loss of ERV DNA methylation and consequent aberrant overexpression of ERVs ${ }^{22-25}$. In particular, treatment of cancer cells with epigenetic inhibitors increases the expression of ERVs and subsequently induces IFN pathway activation ${ }^{14,18,23,26,27}$. Despite this strong evidence that endogenous dsRNAs could induce antiviral innate immune responses in human cells, it has 
28 been not fully understood how normal and malignant cells prevent cellular 'self' dsRNA from

29 being recognized by the cytosolic dsRNA sensors and thereby triggering a potentially

30 detrimental innate immune response.

Human ADARs (adenosine deaminases) are known to be critical regulators of dsRNAs ${ }^{7,28}$.

33 ADAR1 edits adenosine $(\mathrm{A})$ of the double-stranded regions to inosine (I) (known as A-to-I RNA

34 editing), which results in the disruption of dsRNA structures or the retention of edited dsRNAs in

35 the nucleus ${ }^{29,30}$. Also, in the mitochondria, RNA degradosome components SUV3 and

36 polynucleotide phosphorylase (PNPase) prevent the accumulation of mitochondrial double-

37 stranded RNAs ${ }^{9,10,15}$.

DEAD-box RNA helicase 3X (DDX3X) is a member of DEAD-box RNA helicase superfamily

$40 \quad 2^{31,32}$. DDX3X utilizes ATP hydrolysis and RNA helicase activities to unwind RNA duplexes, and

41 participates in the multiple aspects of RNA metabolism, such as transcription, RNA splicing,

42 RNA transport, and initiation of translation ${ }^{33-36}$. DDX3X has been considered as a promising

43 target for anticancer because of its predicted druggability and involvement in tumorigenesis ${ }^{37-43}$.

44 In particular, increased DDX3X levels have been found in primary and metastatic breast cancer,

45 and this high expression has been correlated with worse survival ${ }^{40,44-46}$. Nevertheless, the

46 pathological mechanisms by which DDX3X expression decreases cancer survival remain largely

47 unknown.

Here, we reveal that loss of DDX $3 X$ leads to the aberrant cytosolic accumulation of

50 endogenous dsRNAs in the breast cancer cells, which triggers intrinsic type I IFN production

51 through the activation of cytoplasmic dsRNA sensing pathway along with resultant anti-

52 proliferative effect. Inhibiting DDX3X expression also enhances the antigen presentation on the

53 cancer cells as well as anti-tumor immunity in the syngeneic breast tumor mouse model. Taken 
54 together, these observations suggest that targeting DDX3X could be a novel way of enhancing

55 anti-tumor immunity and thus contribute to combination immunotherapy approaches for patients 56 with breast cancer and other malignancies.

\section{RESULTS}

Loss of DDX3X increases the expression of genes in antiviral innate immune response in breast cancer cells.

High expression of DDX3X mRNA and protein is correlated with worse survival in patients

63 with breast cancer in Kaplan-Meier plot analysis (Extended Data Fig. 1a). To assess the impact

64 of DDX3X on the gene expression in the breast cancer, we stably depleted DDX3X in breast

65 cancer MCF7 cells using a short hairpin RNA (shRNA)-mediated knockdown, and performed a genome-wide transcriptome analysis using a next-generation RNA deep sequencing (Fig. 1a and Extended Data Fig. 1b). Compared to the control MCF7 with shNon-specific RNA (shNS), DDX3X KD (shDDX3X) greatly increased the genes related to antiviral innate immune

71 cells identified the upregulation of type I interferon signaling, antigen presentation pathway

72 (APP), and pattern recognition receptors (PRRs) response to bacteria and virus (Fig. 1b). In the

73 gene-set enrichment analysis (GSEA) of DEGs, the top 5 gene sets co-enriched in the DDX3X

$74 \mathrm{KD}$ cells included type I interferon (IFN), antigen presentation via major histocompatibility

75 complex (MHC) class I, and viral defense response (Fig. 1c). 
80 processing and presentation (APP) such as MHC class molecules (HLA-B, HLA-C, and HLA-

$81 D R A$ ), peptide transporters (TAP1 and TAP2), and immunoproteasome (PSMB8) (Fig. 1d). We

82 observed the similar upregulation of ISGs in the siRNAs-mediated transient DDX3X KD in

83 MCF7 cells (Extended Data Fig. 1c). Knockdown of other DEAD-box helicases such as DDX1

84 or DDX5 did not present a similar induction of ISGs, suggesting the specific role of DDX3X in

85 the regulation of innate immune responses (Fig. 1e and Extended Data Fig. 1d). Similar

86 upregulation of ISGs was validated in DDX3X-depleted breast cancer MDA-MB-453 cells

87 (Extended Data Fig. 1e). This increased ISGs expression suggests the global upregulation of an

88 IFN-driven transcriptional activation in the DDX3X-depleted breast cancer cells.

Type I IFN production and STAT1 pathway are activated in DDX3X-depleted cancer cells.

To determine if DDX3X knockdown (KD) induces IFN production, we measured the levels

92 of the type I IFN (interferon-alpha, $\alpha$; interferon-beta, $\beta$ ) and type II IFN (interferon-gamma, $\gamma$ ) in

93 the culture media of the DDX3X KD cancer cells. DDX3X KD significantly induced the

94 expression and secretion of the type I IFN- $\alpha$ and IFN- $\beta$, but not type II IFN- $\gamma$ in the human and

95 mouse breast cancer cells, including MCF7, MDA-MB-453, and E0771 (Fig. 2a). We also

96 observed the increased production of IFN- $\alpha$ and IFN- $\beta$ in the DDX3X-depleted melanoma cell

$97 \quad$ A375 (Extended Data Fig. 2a).

Canonical type I IFN signaling leads to the transcription of hundreds of IFN-stimulated genes (ISGs) through the activation of Janus kinase-signal transducer and activator of

101 transcription (JAK/STAT) pathway ${ }^{2}$. To determine whether the loss of DDX3X could activate the 102 JAK/STAT pathway, we assessed the components of the STAT pathway. We confirmed that 103 MCF7 and MDA-MB-453 express the IFN receptors and have an intact JAK/STAT pathway in 104 response to type I IFN treatment (Extended Data Fig. 2b,c). DDX3X KD increased the level of 105 the phosphorylated STAT1 and total STAT1 (Fig. 2b). The phosphorylated STAT2 was not 
detected, but DDX3X KD increased total STAT2, which belongs to ISGs (Extended Data Fig.

$1072 \mathrm{~d}$ ). The activated antiviral response often results in the induction of the apoptosis or growth

108 inhibition in the cells ${ }^{2}$. DDX3X KD did not induce apoptosis (Extended Data Fig. 2e), but

109 significantly suppressed the cell proliferation (Fig. 2c and Extended Data Fig. 2f). Taken

110 together, these data suggest that the depletion of DDX3X in breast cancer cells significantly

111 induces cancer cell production of type I IFNs that drives ISG expression through the STAT1

112 pathway, and leads to an anti-proliferative effect.

114 Loss of DDX3X enhances antigen presentation and sensitizes breast cancer cells to IFN

\section{5 treatment.}

116 Next, we observed that IFN- $\alpha$ treatment more dramatically increases the ISG expression in

117 the DDX3X KD than control MCF7 cells, showing that inhibiting DDX3X sensitizes the cancer

118 cells to type I IFN treatment (Fig. 2d). To further determine the functional consequences of the

119 increased IFN- $\alpha$ and IFN- $\beta$ production on the antigen presentation of DDX3X KD cells, we

120 assessed the cell surface expression of MHC class I proteins using flow cytometry. DDX3X-

121 depleted MCF7 cells displayed the significantly enhanced expression of MHC class I proteins

122 (HLA-ABC) (Fig. 2e). Although qRT-PCR analysis showed the increased gene expression of

$123 H L A-D R A$, one of the subunits of MHC class II molecule (Fig. 1d), we did not see the increased

124 MHC II protein complex on the DDX3X KD cells (Extended Data Fig. $2 \mathrm{~g}$ ). We also stably

125 knocked-down DDX3X in the ovalbumin (OVA)-expressing mouse cancer cells, B16-OVA.

126 DDX3X KD increased the presentation of the OVA epitope (SIINFEKL) derived from OVA in

$127 \mathrm{MHC}$ I molecules on the cell surface (Fig. 2f). The OVA peptide presentation was dramatically

128 increased in the DDX3X KD cells by IFN-y stimulation (Fig. 2f). This data indicates that DDX3X

$129 \mathrm{KD}$ enhances the antigen processing as well as antigen presentation via $\mathrm{MHC}$ I on the cancer

130 cells. 


\section{Inhibiting DDX3X triggers the accumulation of endogenous double-stranded RNAs.}

133 Failure to degrade the excess endogenous double-stranded RNAs (dsRNAs) is known to

134 activate the cytoplasmic dsRNA sensors and trigger type I IFN response in the several types of

135 cancer cells ${ }^{3,7,9,12}$. Because DDX3X is an RNA-binding protein, which has an activity for dsRNA

136 unwinding, we speculated that DDX3X could impact the level of endogenous dsRNAs. To

137 measure endogenous dsRNA in the cells, we used a monoclonal anti-dsRNA specific J2

138 antibody (Scion, Hungary), which is widely used to recognize viral and cellular dsRNA of more

139 than 40-bp length with no sequence specificity in animals and plants ${ }^{47-49}$.

First, we validated the suitability of the $\mathrm{J} 2$ antibody to detect endogenous dsRNA in our

142 systems, including immunostaining, dot blot, northwestern blot, and flow cytometry. A dsRNA-

143 specific J2 antibody specifically detected the dsRNA signals in the various cancer cells. The J2

144 signals were sensitive to the dsRNA-specific RNase III or RNase A, but not affected by the

145 treatment of the single-stranded RNA (ssRNA)-specific RNase I or DNase I (Extended Data Fig.

$1463 a, b)$. J2 antibody also recognized the synthetic dsRNA mimic analog, polyinosinic-

147 polycytidylic acid (poly (I:C)) in vitro and in the poly I:C transfected cells (Extended Data Fig. 3c).

148 DNA methyltransferase inhibitors are known to increase the bi-directional transcription of

149 endogenous retroviral elements (ERVs) or repetitive RNA elements, which could increase the

150 RNA duplexes (Extended Data Fig. 3d) ${ }^{18,26,50}$. J2 antibody was able to distinguish the increased

151 level of endogenous dsRNAs in response to the treatment of DNA methyltransferase inhibitor,

152 5-AzaC in the several cancer cells using dot blot and flow cytometry approaches (Extended

153 Data Fig. 3e, f). Collectively, these data validate that the $\mathrm{J} 2$ antibody specifically recognizes the

154 authentic cellular dsRNAs in cancer cells.

156 Next, using the validated anti-dsRNA specific J2 antibody, we investigated the level of 157 endogenous dsRNAs in the DDX3X-depleted breast cancer cells. We found that depletion of 
158 DDX3X substantially increased the level of endogenous dsRNAs in MCF7 cells using

159 immunostaining (Fig. 3a), flow cytometry (Fig. 3b), northwestern blot (Fig. 3c), and dot blot

160 analysis approaches (Fig. 3d and Extended Data Fig. 3g). Enhanced dsRNA signals were also

161 detected in the human and mouse DDX3X KD cancer cells (MDA-MB-453, A375, 4T1, B16F10)

162 (Fig. 3e). Next, We checked the transcription level of the ERVs that were previously identified

$16318,25,26,50$. DDX3X-depleted MCF7 cells showed the increased expression of several ERVs (Fig.

164 3f). Using TAG-aided sense/antisense transcript detection PCR (TASA-TD PCR) that detects

165 the bi-directional expression of ERVs, we validated the dsRNA form of ERVs (Fig. 3g). Similarly,

166 increased ERV expression was detected in the DDX3X-depleted MDA-MB-453 cells (Fig. 3h).

167 HEK293T cells are known to be defective in their ability to produce type I IFN in response to

168 viral RNAs or poly I:C stimulation ${ }^{51}$. There was no induction of ISGs or IFN- $\beta$, but dsRNAs still

169 accumulated in the DDX3X KD HEK293T cells (Extended Data Fig. 3h, i), indicating that dsRNA

170 accumulation is a direct consequence of DDX3X loss, rather than IFN activation. In addition to

171 the J2 antibody, we also confirmed similar results using another dsRNA-specific K1 antibody

172 (Scion, Hungary) ${ }^{48}$ (data not shown). Taken together, these data indicate that DDX3X prevents

173 dsRNA accumulation in the human and mouse cells, suggesting the functional association of

174 DDX3X in regulating the level of endogenous dsRNAs.

Inhibiting DDX3X activates cytoplasmic dsRNA response signaling in cancer cells.

177 Next, we investigated which signaling pathway is responsible for type I IFN production in

178 the DDX3X-KD breast cancer cells. Pattern recognition receptors (PPRs) recognize the

179 intracellular double-stranded DNAs (dsDNAs) or dsRNAs, and subsequently induce type I IFN

180 production ${ }^{1}$. First, we examined cytosolic DNA sensing-mediated innate immune response

181 through STING pathway ${ }^{52}$. There was no activation in cyclic GMP-AMP synthetase (cGAS), a

182 central receptor of cytosolic DNA, and its downstream target, STING (Fig. 4a). This indicates no 
183 involvement of a cytosolic DNA, and that DDX3X KD induces type I IFN in a STING-

184 independent manner.

Because the loss of DDX3X resulted in the accumulation of dsRNAs (Fig.3), we investigated dsRNA-sensing pathways. First, we examined several intracellular dsRNA sensors, including

188 PKR, TLR3, OAS1, RIG-I (DDX58), and MDA5 (IFIH1). The levels of TLR3, phosphorylated

189 PKR, and phosphorylated elF2 $\alpha$ were not changed, indicating that there was no PKR or TLR3

190 activation by DDX3X depletion (Fig. 4b). However, the protein and mRNA expression of MDA5,

191 RIG-I, and OAS1 were significantly increased in the DDX3X KD cells (Fig. 4c and Extended

192 Data Fig. 4a, b). Next, we investigated if there were changes in the transcription factors (IRF3,

193 IRF7, NFאB) that are responsible for IFN- $\beta$ production in DDX3X KD cells. Phosphorylated

$194 \mathrm{NF} \kappa \mathrm{B}$ was consistently increased in the DDX3X-depleted cancer cells (Fig. 4d). DDX3X KD

195 MCF7 cells showed no phosphorylation of IRF3 or IRF7 (Fig. 4d), although MCF7 cells have an

196 intact activation of IRF3 and IRF7 in response to poly I:C treatment (Extended Data Fig. 4c).

197 CRISPR/Cas9-mediated MDA5 knockout significantly diminished the induction of IFN- $\beta$

198 expression by the DDX3X depletion in the MCF7 cells (Fig. 4e and Extended Data Fig. 4d). This

199 suggests that MDA5 is the critical dsRNA sensor to initiate the dsRNA-mediated type I IFN

200 production in the DDX3X-depleted MCF7 cells. Collectively, these data imply that inhibiting

201 DDX3X increases the endogenous dsRNA abundance, which activates the cytosolic dsRNA

202 sensing pathway, mainly the MDA5-NFאB signaling axis, that leads to type I IFN production in

203 breast cancer cells.

205 Type I IFN and dsRNAs signaling pathway are also activated in the DDX3X-null MEF cells.

206 Furthermore, we investigated if the loss of DDX3X could activate dsRNA signaling in mouse

207 embryonic fibroblast (MEF) cells. We generated a $D d x 3 x$ conditional knockout mouse (named 
$208 \mathrm{DDX}^{\mathrm{ff/f}}$ ), in which Ddx3x exons 5 7 were floxed by two loxP sites (Extended Data Fig. 4e, f).

209 MEF cells were isolated from E12.5 embryos by breeding DDX3 $\mathrm{X}^{\mathrm{f} /+}\left(\right.$ or DDX $\left.3 \mathrm{X}^{\mathrm{f} / \mathrm{f}}\right)$ and $\mathrm{DDX} 3 \mathrm{X}^{\mathrm{Y} / \mathrm{f}}$

210 mice, and treated with Cre recombinase expressing adenovirus (Ad-Cre) to induce Cre-

211 mediated $D d x 3 x$ gene deletion. Both hemizygous and homozygous DDX3X-null MEF cells

$212\left(D d x 3 x^{Y / f}\right.$ male and $D d x 3 x^{f / f}$ female $)$ showed the increased total STAT1 and STAT2 levels

213 (Extended Data Fig. 4g). Heterozygous DDX3X knockout ( $D d x 3 x^{f /+}$ female) MEF cells showed

214 the less significant increased STAT1 and no induction of STAT2 level after Ad-Cre treatment

215 (Fig. 4f and Extended Data Fig. 4g). Consistent with the Cre-mediated DDX3X-null MEF cell,

216 shRNA-derived DDX3X knockdown MEF cells also showed the increased level of STAT1 and

217 STAT2, while the non-specific shRNA did not affect the level of STAT1 and STAT2 (Fig. 4g).

218 Consistent with the cancer cells, the loss of DDX3X in the MEF cells also showed the increased

219 phosphorylation of NFKB (Fig. 4f). Importantly, IFN- $\beta$ mRNA expression was increased in the

220 DDX3X-null MEF cells (Fig.4h and Extended Data Fig. 4h). Taken together, these data suggest

221 that DDX3X prevents the activation of cellular dsRNA signaling and type I IFN response in the

222 human cancer cells and mouse embryonic fibroblast (Fig. 4i).

224 DDX3X interacts with cytoplasmic ADAR1 and prevents the buildup of cytoplasmic 225 dsRNA.

226 To understand how DDX3X depletion could activate the cytosolic dsRNA sensors, we first 227 assessed the nuclear-cytoplasmic distribution of dsRNAs in DDX3X-control or -KD MCF7 cells.

228 We isolated total RNAs from the nuclear and cytoplasmic fractions of the DDX3X-control or -KD

229 MCF7 cells, respectively, and applied the isolated RNAs to the dot blot analysis with a dsRNA-

230 specific J2 antibody (Fig. 5a and Extended Data Fig. 5a). Most of dsRNA was found in the

231 nuclear fraction, despite the fact that the amount of RNAs used for dot blot analysis was much

232 higher in the cytoplasmic fraction than the nuclear fraction (Fig. 5a and Extended Data Fig. 5b).

233 DDX3X KD not only increased the level of dsRNA in the nuclear fraction but also substantially 
234 increased the cytoplasmic level of dsRNAs (4.3\% to 19.3\%) (Fig. 5a and Extended Data Fig. 5b).

235 This suggests that DDX3X regulates dsRNAs in both nucleus and cytoplasm. Next, we

236 determined whether this increased cytosolic dsRNAs are the result of mitochondrial dsRNA

237 (mtdsRNA) leaking from the mitochondria. We did not see any increased mitochondrial RNAs in

238 the cytosolic fraction of DDX3X KD cells compared to the control cells, suggesting the

239 accumulated endogenous dsRNAs mainly originated from the genomic DNA, not from the

240 mitochondria (Extended Data Fig. 5c).

steady-state localization is mostly cytoplasmic ${ }^{53,54}$, When CRM1-dependent nuclear export was

244 blocked with the drug leptomycin $\mathrm{B}^{55}$, DDX3X was stuck in the nucleus, and subsequently, the

245 cytoplasmic level of DDX3X (60\% reduction) was decreased (Fig. 5b). Interestingly, when we

246 blocked the nuclear export with leptomycin B, the level of cytoplasmic dsRNAs was increased in

247 the cytoplasm while the nucleus dsRNA level was unchanged (Fig. 5b). This data suggests that

248 the level of cytoplasmic DDX3X is important to maintain the endogenous dsRNAs in the

249 cytoplasm at a minimum level. Next, to further determine whether DDX3X is directly involved in

250 regulating the level or stability of endogenous dsRNAs in cells, we examined if DDX3X is

251 directly associated with endogenous dsRNAs in the cells. We carried out immunoprecipitation to

252 pull-down dsRNA with a dsRNA-specific J2 antibody in the MCF7 cells after treating with

253 formaldehyde to generate cross-links between RNA and protein. DDX3X was co-precipitated

254 with dsRNAs, but not with the control IgG (Fig. 5c). Furthermore, 5-AzaC treatment, which

255 induces the transcription of endogenous dsRNAs, enhanced the binding of DDX3X with dsRNAs

256 (Fig. 5c). This indicates that DDX3X is directly associated with endogenous dsRNAs in the cells.

In particular, DDX3X specifically interacts with ADAR1's two isoforms (110 kDa, ADAR1-

259 p110; 150 kDa, ADAR1-p150) in MCF7 cells (Fig. 5d and Extended Data Fig. 5d). ADAR1 edits 
dsRNA (from Adenosine to Inosine) mostly in the nucleus, and most ADAR1-p110 is found in

261 the nucleus ${ }^{7,56}$. Because DDX3X has been found mainly in the cytoplasm (Extended Data Fig.

$2625 \mathrm{e}$ ), we further dissected the subcellular localization for the interaction between DDX3X and

263 ADAR1. We found that DDX3X interacted with primarily ADAR1-p150 in the cytoplasm (Fig. 5d,

264 right panel). Notably, DDX3X KD alone increased the levels of dsRNA sensors (RIG-I, MDA5),

265 ISGs (IFI44L, OAS1, STAT1), and IFN beta comparable to the effect of ADAR1 KD alone (Fig.

266 5e-g and Extended Data Fig. 5g). Moreover, the double knockdown of ADAR and DDX3X

267 greatly increased the expression of RIG-I, MDA5, other ISGs as well as IFN beta (Fig. 5e-g). To

268 further explore if DDX3X directly impacts on ADAR1-mediated A-to-I dsRNA editing, we used a

269 dual-luciferase reporter for monitoring ADAR-editing efficiency (gift from Dr. Ohman) ${ }^{57}$. ADAR1-

270 mediated dsRNA editing activity was determined as the ratio between luminescence from the

271 Nano luciferase/Firefly luciferase (Extended Data Fig. 5h). We transiently expressed a negative

272 ( $0 \%$ editing), a positive (100\% editing), and an editing reporter (A can be edited to I) vectors,

273 respectively, to validate ADAR1 editing activity in the different parental cell lines (Extended Data

274 Fig. 5i). We found that DDX3X KD alone in MCF7 cells did not impact on the activity of ADAR1-

275 mediated A-to-I editing (Fig. 5h). However, the double knockdown of ADAR1 and DDX3X

276 significantly reduced the dsRNA editing activity relative to ADAR1 KD alone (Fig. 5h). These

277 data indicate that double-inhibition of DDX3X and ADAR1 synergistically activates the cytosolic

278 dsRNA-signaling pathway and an innate immune response in the cancer cells.

280 Low DDX3X level is related to the chronic activation of the innate antiviral immune

281 response in the various cancer cells.

282 To explore DDX3X-dsRNAs-type I IFN signaling axis in other solid cancers, we compared

283 the gene expression profiles in the DDX3X-highly expressing cancer cells (DDX3X ${ }^{\text {hi }}, 253$ cell

284 lines) vs. DDX3X-low expressing cells (DDX3X ${ }^{\text {low }}, 253$ cell lines) of the Cancer Cell Line

285 Encyclopedia (CCLE) database (Extended Data Fig. 6a). The DDX3X expression in both 
286 DDX3X hi and DDX3 $X^{\text {low }}$ groups was not affected by sex or origin of the tumor types (Extended

287 Data Fig. 6b, c). We identified 386 differentially expressed genes (DEGs) in DDX3X ${ }^{\text {hi }}$ vs.

288 DDX3X ${ }^{\text {low }}$ cancer cells (cut off at $p$-value $<0.01$ and fold change $>1.5$ ). Forty-nine genes in the $289 \mathrm{DDX} 3 \mathrm{X}^{\mathrm{hi}}$ cell group and 337 genes in the $\mathrm{DDX} 3 \mathrm{X}^{\mathrm{low}}$ cell group were upregulated, respectively 290 (Fig. 6a).

In the top 5 enriched gene sets identified in DEGs between DDX3X hi vs. DDX3X ${ }^{\text {low }}$, the 294 such as inflammatory response, interferon-a response, and allograft rejection (Fig. 6b). On the 295 other hand, UV response, E2F target, mitotic spindle, and G2M checkpoint were enriched in the 296 DDX3X ${ }^{\text {hi }}$ group (Fig. 6b). In line with the finding that DDX3X inhibition induces the type I IFN 297 and MHC class I expression in the breast cancer cells, the elevated level of MHC class genes, 298 types I IFN (IFNA1, IFNB1, IFNK, IFNE), and proinflammatory cytokines (IL6, IL1) were found in 299 the DDX3X ${ }^{\text {low }}$ cells (Fig. 6c and Extended Data Fig. 6d, e). This CCLE data suggests that DDX3X expression level is inversely correlated with the increased intrinsic type I IFN and innate 301 immune responses in the various cancer types.

We further investigated the functional relationship between DDX3X and ADAR in the 304 endogenous dsRNA-mediated innate immune response in various cancer cells. Previous 305 studies reported that ADAR1 knockdown kills certain cancer cells, and that STING dependently 306 elevated-intrinsic Type I IFN signaling confers a dependency of these cancer cells on ADAR1 $307 \quad 58,59$. We selected ADAR1-dependent (ADAR1 ${ }^{\text {dep }}, 207$ cell lines) and -independent cancer cell 308 (ADAR1 $1^{\text {ind }}, 207$ cell lines) groups from the DRIVE dataset ${ }^{60,61}$ (Extended Data Fig. 6a). As 309 expected, the ADAR $1^{\text {dep }}$ cell group has a high dependency score for the ADAR1 gene (Fig. 6d, 310 top left panel) (score 0 means a minimal impact of knockdown or knockout of a gene for cell 311 survival; score close to 1 means an essential gene for cell survival). ADAR1 ${ }^{\text {dep }}$ cell group also 
312 showed elevated expression of MHC I and type I IFN (Extended Data Fig. 6f). Interestingly, the

313 ADAR1 level was not different in between ADAR $1^{\text {dep }}$ and ADAR $1^{\text {ind }}$ cells, indicating the transcript

314 level of ADAR1 does not impact on the ADAR1 dependency (Fig. 6d, top middle panel).

315 ADAR $1^{\text {dep }}$ cells showed a lower level of DDX3X than the ADAR $1^{\text {ind }}$ cells (Fig. $6 \mathrm{~d}$, top right panel).

316 DDX3X ${ }^{\text {low }}$ group showed a higher ADAR1 dependency than the DDX3X ${ }^{\text {hi }}$ group (Fig. 6d, bottom 317 left panel).

ADAR1-specific DEIG (ADAR1 ${ }^{\text {dep }}$ vs. ADAR1 $\left.1^{\text {ind }}\right)$ and DDX3X-specific DEIG (DDX3X ${ }^{\text {hi }}$ vs.

321 DDX3X $\left.{ }^{\text {low }}\right)$. We performed GO pathway analysis of ADAR1-specific DEIG, DDX3X-specific

322 DEIG, and co-identified DEIGs in both DDX3X and ADAR1, respectively (Fig. 6e and Extended

323 Data Fig. 6a). Among the top 10 enriched GO pathways, overlapping DEIGs from the DDX3X-

324 specific and ADAR1-specific DEIG genes (indicated as a purple color) demonstrated the

325 enrichment of innate immunity, including defense response to virus, type I interferon signaling

326 pathway, and negative regulation of viral life cycle (Fig. 6e). Especially, DDX3X-specific DEIGs

327 (indicated as a red color) are additionally related to genes involved with T cell activation,

328 leukocyte proliferation, and positive regulation of cytokine production (Fig. 6e). We observed

329 similar results in GO pathway analysis with the total $D E G$ s from ADAR $1^{\text {dep }}$ vs. ADAR $1^{\text {ind }}$ and

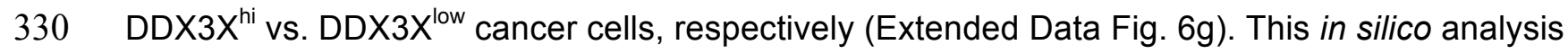

331 of CCLE and DRIVE datasets demonstrates that DDX3X-low cancer cells share similar innate

332 immune-activating signatures with ADAR1-dependent cancer cells, but also display the

333 distinctive gene expression in T cell activation as well as immune cells activation.

335 Inhibition of DDX3X suppresses breast tumor growth and enhances anti-tumor immunity.

336 The expression of the type I IFN and chemokine (ex. CCL5) from the DDX3X-depleted

337 cancer cells could activate effector T cells as well as other immune cells that could further prime 
338 T cells in the tumor. Also, the observation that DDX3X inhibition enhances the efficiency of the

339 cancer cell's antigen presentation through MHC class I prompted us to examine the tumor

340 microenvironment. To investigate the impact of DDX3X inhibition on tumor immune

341 microenvironment, we used the syngeneic mouse breast cancer model. We generated

342 transplantable murine breast cancer cell line 4T1 that express doxycycline (Dox)-inducible

343 DDX3X shRNAs, in which DDX3X level was considerably decreased after Dox treatment

344 (Extended Data Fig. $7 a$ ). $1 \times 10^{5}$ cells of 4 T1 were inoculated into a mammary fat pad of

345 immune-competent BALB/cJ female mouse. When tumors became palpable (20 to $25 \mathrm{~mm}^{3}$ ),

346 randomized groups were treated with doxycycline foods to induce DDX3X knockdown. Tumor

347 growth was monitored with calipers every three days. We found that inhibiting DDX3X

348 substantially suppressed the 4T1 murine breast primary tumor growth, tumor volume, tumor

349 weight, and metastatic spread to the lung in the BALB/cJ syngeneic mouse model (Fig. 7a-c

350 and Extended Data Fig. 7b). The similar tumor growth inhibition by DDX3X depletion was also

351 confirmed in the 4T1 tumor-bearing stable DDX3X KD in BALB/cJ female syngeneic mouse

352 model (Extended Data Fig. 7c, d).

354 Flow cytometry analysis of the DDX3X-depleting 4T1 tumors revealed the significantly

355 increased tumor infiltration of T cells and dendritic cells (DCs) (Fig. 7d, e). To further identify the

356 T cell subsets, we assessed CD4+ helper and CD8+ cytotoxic T cells. The proportions of

357 cytotoxic CD8+ T cells in the DDX3X-depleted tumor was increased, whereas the proportions of

358 helper CD4+ T cell was present at a similar level between DDX3X-KD and -control tumor (Fig.

359 7f). Intratumoral CD8+ T cells in the DDX3X inducible-KD 4T1 tumors displayed the markedly

360 increased expression of the effective cytotoxic CD8+ T cells markers, CD69 and CD44 (Fig. 7g).

361 Similarly, significantly increased tumor infiltration of CD3+CD8+CD69+ T cells,

362 CD3+CD8+CD44+ T cells, macrophage, and DCs was found in the DDX3X stable-KD 4T1

363 tumors (Extended Data Fig. 7e-g). 
To obtain further insight within the tumors, we performed immunohistochemistry on the paraffin-embedded tumor sections. Consistent with the flow cytometry results, the increased

367 CD8+ T cell infiltration was observed in the DDX3X-depleted tumors (Fig. 7h, i). Cytotoxic 368 lymphocyte-secreted a pore-forming perforin, which shows tumor lytic functions, was also 369 augmented in the DDX3X-depleted tumors (Fig. 7h). DDX3X-depleted tumors exhibited the 370 decreased Ki67 proliferation marker (Fig. 7h). This in vivo result revealed that inhibition of

371 DDX3X not only activates the tumor cell-intrinsic type I IFN response but also changes the 372 tumor immune microenvironment resulting in increased tumor-infiltrating cytotoxic CD8+ T cells

373 and DCs. Collectively, these data suggest that inhibiting DDX3X in tumors enhances the antigen

374 presentation and the secretion of cytokines from cancer cells per se, which could induce

375 enhanced anti-tumor immunity in the tumor microenvironment (Extended Data Fig. 7h).

\section{DISCUSSION}

The findings in this study provide insights for the potential molecular mechanism of how DDX3X could regulate the cellular dsRNA homeostasis. Most endogenous dsRNAs are known to be located in the nucleus ${ }^{7}$ or mitochondria ${ }^{15}$, so that cells prevent these dsRNAs from being exposed to the cytosolic dsRNA sensors and triggering a potentially detrimental innate immune responses. The nuclear-cytoplasmic dsRNA distribution data indicates that a small fraction of dsRNAs is present in the cytoplasm in the homeostatic condition without inducing an immune response, but the depletion of DDX3X substantially increases the level of cytoplasmic dsRNAs

385 (Fig. 5a). This suggests that unedited or partially edited genomic dsRNAs or mitochondrial 386 dsRNAs could be transported to the cytoplasm ${ }^{24,62}$ and DDX3X may be important to maintain 387 the endogenous dsRNAs in the cytoplasm at a minimum level. There are several possibilities of 388 how DDX3X could be involved in cellular dsRNA regulation. DDX3X could directly regulate the 389 level or the stability of dsRNAs by unwinding the dsRNA structure. Alternatively, with the feature 
390 of a low complexity-intrinsically disordered region (IDR) in the $\mathrm{N}$ - and C-terminal sequences of

$391 D^{2}{ }^{2}{ }^{63-66}$, it is possible that DDX3X sequesters dsRNAs within liquid-liquid phase-separations

392 in the cytoplasm (e.g. small droplets or stress granules) until further degradation or modification

393 of dsRNAs. Future questions that will need to be addressed are how ATP-, RNA-binding

394 domains, and IDRs of DDX3X are associated with its dsRNA regulating function.

than single knockdown of each gene in activation of cytosolic dsRNA signaling pathway and

398 innate immune response in the breast cancer cells (Fig. 5e-g). This suggests that DDX3X and

399 ADAR1 may be acting independently on dsRNAs. Interestingly, depletion of DDX3X also

400 inhibited ADAR1-mediated dsRNA editing activity when ADAR1 was depleted (Fig. 5h).

401 Therefore, it is also possible that DDX3X could participate in ADAR1-mediated dsRNA editing.

402 Because of the A-form structure of dsRNA, which includes a deep major groove ${ }^{67}$, it is difficult

403 for dsRNA-binding proteins like ADARs to recognize the dsRNA efficiently ${ }^{12}$. Indeed, ADAR

404 cannot bind and edit the dsRNA helices that are buried in a tertiary structure ${ }^{7}$. As an RNA

405 helicase, DDX3X could promote efficient ADAR-mediated dsRNA editing by unwinding the

406 complex three-dimensional RNA structures and exposing dsRNA extension to ADAR1. Or

407 DDX3X could facilitate other endo- or exonucleases for their processing or degradation of

408 dsRNAs. To better understand the molecular mechanism of DDX3X-mediated dsRNA

409 regulation, it will be important to identify the dsRNA species that are directly regulated by

410 DDX3X in future study. Also, genome-wide sequencing analysis will be required to determine

411 the direct impact of DDX3X on A-to-I dsRNA editing efficiency in the cells.

413 The present study additionally provides insight for a deeper understanding of the molecular

414 mechanisms underlying the complex role of DDX3X in human innate immunity and cancer.

415 DDX3X has been linked to viral replication and considered to promote antiviral response ${ }^{68-71}$. In 
416 contrast, some viruses like HIV or HCV utilize DDX3X for their replication, suggesting that

417 DDX3X could be a pro- or anti-viral factor ${ }^{43,72-74}$. In particular, previous findings suggest DDX3X

418 as a positive regulator of IFN production through the direct association with antiviral signaling

419 pathways ${ }^{69,70,73,75}$. In contrast to these findings, our study demonstrates that DDX3X prevents

420 type I IFN production against endogenous dsRNAs by maintaining the homeostasis of

421 endogenous dsRNAs. We note that the previous studies have been carried out in the cells after

422 infection with virus, pathogens, or treatment with the viral mimicking synthetic dsRNAs, whereas

423 the present findings are based on normal or malignant cells in the steady-state condition.

424 Recently, Szappanos et.al showed that DDX3X is an essential for innate immunity against the

425 infection of pathogenic bacteria, but DDX3X is also important for hematopoiesis and keeping

426 maintenance of immune cells in mice, with the most profound effects observed in lymphocytes

427 and splenic NK cells ${ }^{76}$. Most recently, Samir et.al showed a role for DDX3X in driving NLRP3

428 inflammasome and stress granule assembly. They showed that DDX3X interacts with NLRP3

429 and is required for NLRP3 inflammasome activation in bone-marrow-derived macrophages

430 stimulated with lipopolysaccharide ${ }^{77}$. Given the previous and our current findings, we propose

431 that DDX3X may have a "dual effect" on immune activity in humans (anti-autoimmunity and anti-

432 viral activity). Although more detailed studies are required to mechanistically define antiviral

433 activity and anti-autoimmunity against cellular dsRNAs of DDX3X, the current study proposes

434 the complex role of DDX3X in the dsRNA biology network and antiviral immunity. In the future, it

435 will be important to investigate how different RNA viruses utilize this potential dual role of

436 DDX3X for their replication. Our conditional DDX3X knockout mouse could be a useful in vivo

437 tool to systematically define the role of DDX3X.

439 Loss of ADAR1 increases cellular dsRNAs that trigger the type I IFN responses in the

440 various tumor cells and affording increased sensitivity to immunotherapy ${ }^{17,78,79}$. Recently, Liu et

441 al. reported that cancer cells have chronic type I IFN activation and increased ISG signature 
442 triggered by a STING-dependent pathway, rendering them sensitive to ADAR1 loss ${ }^{58}$. The

443 malignant cells may have elevated levels of dsRNAs due to the loss of suppressive epigenetic

444 modifications in repetitive elements, genomic instability, or mitochondrial damage due to

445 metabolic stress, which would increase dsRNA burden in the cells ${ }^{12,80}$. Therefore, cancer cells

446 with the high dsRNAs load could rely more on the active cellular dsRNA regulatory mechanism

447 to avoid the activation of innate immune signaling as well as to escape them from immune

448 surveillance. CCLE analysis results suggest that DDX3X-dsRNAs-type I IFN response axis is

449 not restricted to the specific type of cancer such as breast cancer, but rather depends on the

450 endogenous dsRNAs expression burden or intact innate immune systems of the cancer cells. In

451 GO analysis of DEGs, both ADAR and DDX3X are related to the strong upregulation of antiviral

452 immune signatures including type I interferon signaling pathway and innate immune response,

453 suggesting that DDX3X and ADAR1 are possibly functionally linked to each other in various

454 cancer cells. Notably, the DDX3X expression level is inversely correlated with the immune cell

455 activation, including $\mathrm{T}$ cell activation, lymphocyte proliferation, and regulation of the immune

456 effector process (Fig. 6e). This suggests that the diverse dsRNA regulatory mechanism could

457 induce a different impact on the tumor immune microenvironment.

459 Despite an established benefit of type I IFN in cancer therapy, the exogenous administration

460 of type I IFN has shown systemic adverse effects and at best modest antitumor efficacy, which

461 has reduced its use ${ }^{81}$. Recent findings of endogenous nucleic acid-induced tumor cell-intrinsic

462 type I IFN pathway gives new insights for enhancing the therapeutic effect of type I IFN ${ }^{81,82}$.

463 Tumor cell-intrinsic type I IFN response has been achieved through the intracellular dsRNA

464 transcription by using a CDK4/6 inhibitor ${ }^{16}$ or DNA methylation inhibitors ${ }^{18,26,83}$. Also, cellular

465 dsRNA accumulation has been induced at the post-transcriptional level by inhibiting ADAR1 ${ }^{17}$,

466 LSD1 ${ }^{14}$, or PNPT1 ${ }^{15}$. Our data shows that inhibiting DDX3X enhances cancer cell-intrinsic type

467 I IFN response, antigen presentation on the cancer cells, and the antitumor activity in tumors. In 
468 particular, inhibiting DDX3X increased not only tumor-infiltrated cytotoxic CD8+T cells, but also

469 DCs. CD8+ T cells recognize tumor-associated antigens presented on MHC I by their

470 expressed T cell receptor (TCR) to remove tumor cells and, DCs are required to cross-present

471 exogenous tumor-associated antigens onto $\mathrm{MHC}$ I to prime CD8+ T cells ${ }^{84}$. Furthermore,

472 DDX3X depleted-OVA expressing cancer cells showed the increased ability of antigen

473 presentation onto MHC I. These data show that inhibiting DDX3X could enhance the elimination

474 of cancer cells by CD8+ T cells directly and DCs indirectly. Increased tumor cell antigen

475 presentation coupled with anti-tumor T-cell responses suggests that immune checkpoint

476 blockade might further enhance the efficacy of DDX3X inhibition.

478 In conclusion, the present study identifies a role of DDX3X in regulating the endogenous 479 dsRNAs homeostasis in human and mouse malignant cells as well as MEFs. DDX3X maintains 480 the cytosolic endogenous dsRNAs at a low level, but the loss of DDX3X leads to the aberrant 481 accumulation of cellular dsRNAs, which activates cytoplasmic dsRNA sensors and triggers type 482 I interferon signaling in the breast cancer cells. We further show that inhibiting DDX3X restores 483 cancer immunity and enhances the antitumor activity by inducing the dsRNA-derived type I IFN 484 response in breast tumors, which may lead to novel agents targeting DDX3X for combinatory 485 immunotherapy. 


\section{ACKNOWLEDGEMENTS}

We thank Michael B. Atkins and Xiongbin Lu for a critical reading of the manuscript. We thank Marta Catalfamo, Astrid Haase, and Pavol Genzor for a helpful scientific discussion. We thank Marie Öhman for the kind gift of ADAR1 editing vectors. We thank Charles G. Drake and Mark Smyth for sharing B16-OVA cells. We thank Jing Wang and Xiaogang Zhong for helping the RNA sequencing analysis. This work was in part supported by an $\mathrm{NIH} / \mathrm{NCl}$ Pathway to Independence Award (K99/R00 CA197487).

\section{AUTHOR CONTRIBUTIONS}

Conceptualization, C.H and J.C.; Methodology, M.S.C., and Y.S.; Investigation, H.C., J.K., J.S., and C.H.; Writing - Original Draft, H.C. and J.K.; Writing - Review \& Editing, C.H. J.C.; Funding Acquisition, C.H.; Supervision, C.H.

\section{DECLARATION OF INTERESTS}

The authors declare no competing interests. 


\section{EXPERIMENTAL PROCEDURES}

\section{Cell culture and generation of stable cell lines}

MCF7, MDA-MB-453, MDA-MB-231, A375, B16F10, 4T1, E0771, and HEK293T cell lines were obtained from the American Type Culture Collection and cultured under standard conditions specified by the manufacturer. B16-OVA cells were kindly provided by Dr. Charles G. Drake (Columbia University Medical Center, NY, USA). The cells were maintained in DMEM medium supplemented with $10 \%$ fetal bovine serum, $1 \%$ penicillin/streptomycin, and $250 \mu \mathrm{g} / \mathrm{ml} \mathrm{G} 418$ (Invitrogen) at $37^{\circ} \mathrm{C}$ with $5 \% \mathrm{CO}_{2}$. All the cell lines were tested negative for mycoplasma using the Mycoplasma Detection kit (Lonza). For generation of stable DDX3X knockdown cell lines, lentiviral pGIPZ, inducible TRIPZ, and SMARTvector (Inducible Mouse Ddx3x shRNA) were obtained from Dharmacon. The following sequences are targeted: GIPZ Lentiviral Human DDX3X shRNA \#1_Clone Id_V2LHS_228965: 5'-TAAATCTGACTCAAGATGG-3', GIPZ Lentiviral Human DDX3X shRNA \#2_Clone Id_V3LHS_301003: 5'-

GTACTGCCAACTCTCTCGT-3', GIPZ negative (non-targeting or non-silencing) shRNA control _Catalog ID_RHS4346, TRIPZ Inducible Lentiviral shRNA Human DDX3X \#1_Clone Id_V3THS_301003: 5'-GTACTGCCAACTCTCTCGT-3', TRIPZ Inducible Lentiviral shRNA Human DDX3X \#2_Clone Id_V2THS_228965: 5'-TAAATCTGACTCAAGATGG-3', SMART vector Inducible Mouse Ddx3x PGK-TurboRFP shRNA \#1_Clone Id_V3SM11253-232377132: 5'-TCCCTCTTGAATCACCCCG-3', SMARTvector Inducible Mouse Ddx3x PGK-TurboRFP shRNA \#2_Clone Id_V3SM11253-232987995: 5'-TGCACTGCCAATTCTCTCG-3'.

Recombinant lentiviral particles were produced using a protocol provided by the manufacturer (Addgene). In brief, $2 \mu \mathrm{g}$ of shRNA-encording vector DNA, $1.5 \mu \mathrm{g}$ of psPAX2 (packaging vector) and pMD2.G (VSVG envelope vector) vectors were transfected into HEK293T cells in $94 \mathrm{~mm}^{2}$ dish using TransIT-LT1 Transfection Reagent (Mirus). The supernatants containing virus particles was collected at 72 hours after transfection. Filtered viral supernatants added in the growth medium in the presence of polybrene $(8 \mathrm{mg} / \mathrm{ml})$. To establish stale KD cell lines, cells 
were selected 48 hours after viral infection using $2 \mathrm{mg} / \mathrm{ml}$ of puromycin and collected single colonies and propagated. Knockdown efficiency was validated by western blotting (protein) and qRT-PCR (mRNA). The IFIH1 gene was deleted using a MDA5 (IFIH1) Human Gene Knockout Kit (OriGene, KN415661) according to the manufacturer's instructions with target sequences (5'CTGGATGTACATTTTCACCC-3').

\section{Immunohistochemistry and immunofluorescence.}

For immunohistochemistry, paraffin embedded tumor samples and sections were prepared as previously described ${ }^{85}$. Samples were incubated with primary antibodies which were CD8 (\#98941, Cell Signaling Technology), Ki-67 (\#12202, ell Signaling Technology), and Perforin (\#31647, Cell Signaling Technology) for overnight at $4^{\circ} \mathrm{C}$. Sections were incubated with biotinylated goat anti-rabbit IgG (PK-4000, Vector laboratories) followed by washing three times with TBS-T (0.05\% Tween-20). After washing, sections were incubated with VECTASTAIN ABC Reagent (PK-4000, Vector laboratories) for 30 minutes and then developed using a DAB peroxidase substrate kit (ImmPACT DAB, SK-4105, Vector laboratories). Sections were counterstained with hematoxylin (3801570, Leica). For immunofluorescence, cells were plated on glass coverslips. Cells were washed with PBS for two times and fixed with $4 \%(v / v)$ formaldehyde in PBS at room temperature for 15 minutes and permeabilized with $0.25 \%$ TritonX100 in PBS for 20 minutes at room temperature. J2 antibody was diluted in PBS (1: 100) and incubated at $4^{\circ} \mathrm{C}$ for overnight. Cells were washed with PBS for three times and incubated with rat anti-mouse IgG2a conjugated with APC (407109, BioLegend) for 1 hour at room temperature. Imaging was performed with a Leica SP8 confocal microscope. For nuclease treatment, cells were treated with enzymes at $37^{\circ} \mathrm{C}$ for 30 minutes before fix the cells. Following enzymes were used: RNase I (50 U/ml), RNase A (10 $\mu \mathrm{g} / \mathrm{ml})$, RNase III (20 U/ml) in PBS containing $5 \mathrm{mM}$ $\mathrm{MgCl}_{2}$ 


\section{Western blot}

Cells were washed with ice-cold PBS twice and aspirated residual medium completely. Cell lysis buffer (\#9803, Cell Signaling Technology, composition: $20 \mathrm{mM}$ Tris-HCl (pH 7.5), $150 \mathrm{mM} \mathrm{NaCl}$, $1 \mathrm{mM} \mathrm{Na}{ }_{2}$ EDTA, $1 \mathrm{mM}$ EGTA, $1 \%$ Triton, $2.5 \mathrm{mM}$ sodium pyrophosphate, $1 \mathrm{mM} \beta$ glycerophosphate, $1 \mathrm{mM} \mathrm{Na3VO4,} 1 \mu \mathrm{g} / \mathrm{ml}$ leupeptin, 1mM PMSF, and protease inhibitor cocktail (87785, Thermo Fisher)) was added to cell dishes and scraped on ice. Cell lysates were keep on ice for 30 minutes and centrifuged at $13,200 \mathrm{rpm}$ for 10 minutes at $4^{\circ} \mathrm{C}$. Protein concentrations were measured by Bio-Rad protein assay (\#5000006, Bio-Rad) and equal volume and quantity of protein samples were made by addition of $4 \mathrm{x}$ Laemmli Sample Buffer (\#1610747, Bio-Rad) containing 2-merchatoethanol (\#1610710, Bio-Rad) and boiling at $95^{\circ} \mathrm{C}$ for 10 minutes. Protein samples were subjected to SDS-PAGE and transferred to polyvinylidene difluoride membranes (\#1620177, Bio-Rad). The membranes were blocked with 5\% skim milk in TBS-T $(0.1 \%$ Tween-20) for 30 minutes at room temperature and incubated with indicated primary antibodies at $4^{\circ} \mathrm{C}$ overnight. On the next day, membranes were washed and incubated with appropriate peroxidase-conjugated secondary antibodies for 1 hour at room temperature. Following several washes, ECL (WBKLS0500, Millipore or \#1705061, Bio-Rad) was applied for membrane development. Following antibodies were used: rabbit anti-DDX3 (A300-474A, Bethyl Laboratories), mouse anti-DDX3 (sc-365768, Santa Cruz), rabbit anti-DDX1 (A300-521A, Bethyl Laboratories), rabbit anti-DDX5 (A300-523A, Bethyl Laboratories), rabbit anti-ADAR1 (A300884A, Bethyl Laboratories), mouse anti-ADAR1 (sc-271854, Santa Cruz), mouse anti- $\beta$-Actin (sc-4777, Santa Cruz), rabbit anti-phospho-Stat1 (Tyr701) (\#9167, Cell Signaling Technology), rabbit anti-Stat1 (\#14994, Cell Signaling Technology), rabbit anti-phospho-Stat2 (Tyr690) (\#4441, Cell Signaling Technology), rabbit anti-Stat2 (\#72604, Cell Signaling Technology), rabbit anti-RIG-I (\#4200, Cell Signaling Technology), rabbit anti-MDA-5 (\#5321, Cell Signaling Technology), rabbit anti-OAS1 (\#14498, Cell Signaling Technology), rabbit anti-phospho-PKR (Thr451) (\#07-886, Millipore), mouse anti PKR (sc-6282, Santa Cruz), rabbit anti-phospho- 
elF2a (Ser51) (\#9721, Cell Signaling Technology), rabbit anti-elF2 $\alpha$ (\#9722, Cell Signaling Technology), rabbit anti-cGAS (\#15102, Cell Signaling Technology), rabbit anti-phospho-STING (Ser366) (\#85735, Cell Signaling Technology), rabbit anti-STING (\#13647, Cell Signaling Technology), rabbit anti-phospho-NF-kB p65 (Ser536) (\#3033, Cell Signaling Technology), rabbit anti-NF-kB p65 (\#8242, Cell Signaling Technology), rabbit anti-phospho-IRF-3 (Ser396) (\#29047, Cell Signaling Technology), rabbit anti-phospho-IRF-7 (Ser477) (\#12390, Cell Signaling Technology), rabbit anti-Toll-like Receptor 3 (\#6961, Cell Signaling Technology), mouse anti-Lamin A/C (sc-376248, Santa Cruz), mouse anti-alpha tubulin (ab7291, Abcam), rabbit anti-Drosha (A301-886A, Bethyl Laboratories), mouse anti-FLAG M2 (F1804, Sigma), mouse anti-IFN $\gamma$ R $\alpha$ (sc-12755, Santa Cruz), mouse anti-IFN $\alpha \beta R \alpha$ (sc-7391, Santa Cruz).

\section{Immunoprecipitation}

Cells were lysed in immunoprecipitation buffer (1\% NP-40, $50 \mathrm{mM}$ Tris- $\mathrm{HCl}, 500 \mathrm{mM} \mathrm{NaCl}$ and 5 mM EDTA) containing protease inhibitor cocktails (87785, Thermo Fisher) on ice for 30 minutes. Cell lysates $(800 \mu \mathrm{g})$ were incubated with $3 \mu \mathrm{g}$ of antibodies or normal $\operatorname{lgG}$ at $4^{\circ} \mathrm{C}$ overnight with rotary agitation. For immunoprecipitation of DDX3X or ADAR1, anti-DDX3X (sc365768, Santa Cruz) or anti-ADAR1 (sc-271854, Santa Cruz) was used, respectively. Protein G agarose beads (11243233001, Roche) were added to the lysates and incubated for additional 4 hours at $4^{\circ} \mathrm{C}$ with rotary agitation. The IP Beads were washed in immunoprecipitation buffer three times for 10 minutes each and completely removed residual buffer and boiled in SDS loading buffer for 10 minutes at $95^{\circ} \mathrm{C}$ for western blot analysis. For $\mathrm{J} 2$ immunoprecipitation, cells were fixed with $1 \%$ formaldehyde prior to lysis cells. Immunoprecipitation was performed with J2 antibody (10010200, Scicons) and normal IgG, respectively and coupled to the protein G agarose beads. 


\section{J2 dot blot and northwestern blot}

Total cellular RNA or the RNA of cytoplasmic and nucleus fractions were extracted using the manufacturer's protocol of TRIzol reagent (15596026, Thermo Scientific) or TRIzol LS Reagent (10296010, Thermo Scientific), respectively. RNA concentration was measured by the Nonodrop. For dot blot, purified RNA was dotted on PVDF membrane following pre-dotted site using methanol. The membrane was dried on table for overnight. For northwestern blot, purified RNA was electrophoresed in non-denaturing $2 \%$ agarose TBE gel and then gel was blotted onto an Hybound-N nylon membrane (RPN303N, GE Healthcare) by semidry electroblotting in $0.5 \mathrm{X}$ TBE buffer for 2 hours at $200 \mathrm{~mA}$. The membrane was blocked in $5 \%$ skim milk in TBS-T $(0.1 \%$ Tween-20). J2 antibody was incubated for overnight at $4^{\circ} \mathrm{C}$ and then secondary antibody was incubated for 1 hour at room temperature. ECL was applied for the membrane visualization (WBKLS0500, Millipore or \#1705061, Bio-Rad).

\section{qRT-PCR}

Total cellular RNA or the RNA of cytoplasmic and nucleus fractions were extracted using the manufacturer's protocol of TRIzol reagent (15596026, Thermo Scientific) or TRIzol LS Reagent (10296010, Thermo Scientific), respectively. RNA concentration was measured by the Nonodrop and $1 \mu \mathrm{g}$ of total RNA was used to generate cDNA with the High Capacity cDNA Reverse Transcription Kits (4368814, Thermo Scientific). Quantitative reverse transcription PCR (qRT-PCR) of DDX3X, IFI44L, IFIT2, IFIT3, ISG15, STAT1, OAS1, OAS2, MX1, TAP1, TAP2, PSMB8, HLA-A, HLA-B, HLA-C, HLA-DRA, RIG-I, MDA5, and IFNB1 was performed using TaqMan Fast Advanced Master Mix (4444557, Applied Biosystems). qRT-PCR of envK1-6, enVV1, MER21C, MER57B1, ERVF, ERVFb1, ERV9-1, MATA10, MLT2B4, ENV-MER34, and ERV-Fb1 was performed using SYBR Green master mix (A25742, Applied Biosystems). All qRT-PCR was performed on a QuantStudio 3 Real-Time PCR system and software (Applied Biosystems). GAPDH or $18 \mathrm{~s}$ were used as reference genes. All qRT-PCR assays were carried 
out in triplicate and then repeated with new cDNA synthesis. Primer information is summarized in Supplemental Table 2.

\section{Animal experiments}

Female BALB/cJ mice (6-8 weeks old) were purchased from Jackson Laboratories. All studies were approved and supervised by the Institutional Animal Care and Use Committee at Georgetown University. To generate syngeneic mouse mammary tumor model, $1 \times 10^{5} 4 \mathrm{~T} 1$ inducible DDX3X knockdown cells were implanted into mammary fat pad after mixed 1:1 by volume with matrigel (Corning). Before inducing of DDX3X knockdown, mice were divided into control and DDX3X knockdown groups of equal average tumor volume. Doxycycline water (5\% sucrose with $2 \mathrm{mg} / \mathrm{ml}$ of doxycycline (Sigma) was provided to DDX3X knockdown group to induce knockdown of DDX3X from 1 week after cell injection. The doxycycline water was changed every other day. Tumor size was measured every 3-5 days by caliper. The tumor volume calculated using the formula: Volume $=\left(\right.$ Width $^{2} \times$ Length $) / 2$.

\section{Flow cytometry}

For tumor infiltrating leukocyte flow cytometry, tumors $(0.5 \mathrm{~g})$ were mechanically disrupted by chopping and then chemically digested using Tumor Dissociation Kit (130-096-730, Miltenyi Biotec) and gentleMACS Dissociator (130-096-427, Miltenyi Biotec) according to the manufacturer's instructions. Isolated tumor cells were lysed red blood cells (10-548E, Lonza) and blocked Fc receptors with anti-CD16/32 (101319, BioLegend) for 20 minutes on ice and then stained with appropriate antibodies for 1 hour on ice. Before flow cytometry analysis, cells were stained with Zombie NIR Fixable Viability Kit (423105, BioLegend) to distinguish live or dead cells. For OVA or HLA class expression on cell surface, cells were detached with 2 mM EDTA in PBS and then washed twice using PBS before staining. Cells were stained with appropriate antibodies for 30 minutes on ice and then washed twice before flow cytometry 
analysis. The following antibodies were used: APC conjugated anti-mouse $\mathrm{H}-2 \mathrm{~Kb}$ bound to SIINFEKL (141605, BioLegend) and mouse IgG1, K Isotype control (555751, BD Pharmingen), APC conjugated anti-human HLA-DR (307609, BioLegend) and mouse IgG2a isotype control (407109, BioLegend), and APC conjugated Anti-Human HLA-ABC (562006, BD Pharmingen) and mouse IgG1, k Isotype control (555751, BD Pharmingen). For J2 flow cytometry, cells were detached with 2 mM EDTA in PBS and then washed twice using PBS. Cells were fixed with $1 \mathrm{x}$ fixation buffer (424401, BioLegend) for 20 minutes at room temperature. After washing, cells were permeabilized with $0.1 \%$ Triton-X-100 in PBS for 15 minutes followed by incubation in $1 \%$ BSA in PBS for 30 minutes. Cells were stained with J2 antibody (10010200, Scicons) or mouse IgG2a isotype control (401501, BioLegend) for 1 hour at room temperature followed by antimouse IgG2 conjugated with APC was stained for secondary antibody. Flow cytometry were performed with a FACSCalibur (BD Biosciences) and data were analyzed using FCS express 6.

\section{CCLE data analysis}

Gene expression and gene effect data were obtained from the CCLE and DeMap portal (http://doi:10.6084/m9.figshare.11384241.v2). Cell lines with fibroblast and hematopoietic lineage expression profile were excluded from the analysis. DDX3X high expressing (DDX3X ${ }^{\text {hi }}$ ) cells and DDX3X low expressing (DDX3 $\left.\mathrm{X}^{\text {low }}\right)$ cells were selected by DDX3X expression level (top $25 \%$ and bottom $25 \%$, respectively). ADAR1 dependency data was downloaded from DeMap portal. Gene effect score is obtained by large scale RNAi screening. A lower score means that a gene is more likely to be dependent in a given cell line. We used gene dependency score as a reverse value of gene effect score. ADAR1 dependent- (ADAR1 ${ }^{\text {dep }}$ ) and independent- $\left(A D A R 1^{\text {idp }}\right)$ groups were divided by ADAR1 gene dependency (top $25 \%$ and bottom $25 \%$, respectively). RNA-seq data from selected groups was normalized with the voom method and differential expression determined by limma ${ }^{86}$. MHC core scores were calculated with mean absolute deviation modified Z-score mRNA expression data of in CCLE. The score 
was defined as the mean Z-score of all MHC class genes in each group. GSEA analysis was performed using fgsea function ${ }^{87}$ and KEGG and GO term enrichment analysis on expression data was conducted using clusterProfiler function from R Bioconductor package ${ }^{88}$. To select immune-related genes, differentially expressed genes in each group were compared with innateDB (https://www.innatedb.com) ${ }^{89}$. Differentially expressed immune-related gene lists were subjected as an input for GO enrichment analysis 


\section{REFERENCE}

1. Schlee, M. \& Hartmann, G. Discriminating self from non-self in nucleic acid sensing. Nat Rev Immunol 16, 566-580 (2016).

2. Ivashkiv, L.B. \& Donlin, L.T. Regulation of type I interferon responses. Nat Rev Immunol 14, 36-49 (2014).

3. Uggenti, C., Lepelley, A. \& Crow, Y.J. Self-Awareness: Nucleic Acid-Driven Inflammation and the Type I Interferonopathies. Annu Rev Immunol 37, 247-267 (2019).

4. Gong, T., Liu, L., Jiang, W. \& Zhou, R. DAMP-sensing receptors in sterile inflammation and inflammatory diseases. Nat Rev Immunol 20, 95-112 (2020).

5. Cao, X. New DNA-sensing pathway feeds RIG-I with RNA. Nat Immunol 10, 1049-1051 (2009).

6. Bhate, A., Sun, T. \& Li, J.B. ADAR1: A New Target for Immuno-oncology Therapy. Mol Cell 73, 866-868 (2019).

7. Reich, D.P. \& Bass, B.L. Mapping the dsRNA World. Cold Spring Harb Perspect Biol 11(2019).

8. Portal, M.M., Pavet, V., Erb, C. \& Gronemeyer, H. Human cells contain natural doublestranded RNAs with potential regulatory functions. Nat Struct Mol Biol 22, 89-97 (2015).

9. Linder, A. \& Hornung, V. Mitochondrial dsRNA: A New DAMP for MDA5. Dev Cell 46, 530-532 (2018).

10. Riley, J.S. \& Tait, S.W. Mitochondrial DNA in inflammation and immunity. EMBO Rep 21, e49799 (2020).

11. Katayama, S., et al. Antisense transcription in the mammalian transcriptome. Science 309, 1564-1566 (2005).

12. Hur, S. Double-Stranded RNA Sensors and Modulators in Innate Immunity. Annu Rev Immunol 37, 349-375 (2019).

13. Ahmad, S., et al. Breaching Self-Tolerance to Alu Duplex RNA Underlies MDA5Mediated Inflammation. Cell 172, 797-810 e713 (2018).

14. Sheng, W., et al. LSD1 Ablation Stimulates Anti-tumor Immunity and Enables Checkpoint Blockade. Cell 174, 549-563 e519 (2018).

15. Dhir, A., et al. Mitochondrial double-stranded RNA triggers antiviral signalling in humans. Nature 560, 238-242 (2018).

16. Goel, S., et al. CDK4/6 inhibition triggers anti-tumour immunity. Nature 548, 471-475 (2017).

17. Ishizuka, J.J., et al. Loss of ADAR1 in tumours overcomes resistance to immune checkpoint blockade. Nature 565, 43-48 (2019).

18. Chiappinelli, K.B., et al. Inhibiting DNA Methylation Causes an Interferon Response in Cancer via dsRNA Including Endogenous Retroviruses. Cell 162, 974-986 (2015).

19. Lander, E.S., et al. Initial sequencing and analysis of the human genome. Nature 409, 860-921 (2001).

20. Tokuyama, M., et al. ERVmap analysis reveals genome-wide transcription of human endogenous retroviruses. Proc Natl Acad Sci U S A 115, 12565-12572 (2018).

21. Bannert, N. \& Kurth, R. Retroelements and the human genome: new perspectives on an old relation. Proc Natl Acad Sci U S A 101 Suppl 2, $14572-14579$ (2004).

22. Flemr, M., et al. A retrotransposon-driven dicer isoform directs endogenous small interfering RNA production in mouse oocytes. Cell 155, 807-816 (2013).

23. Jones, P.A., Ohtani, H., Chakravarthy, A. \& De Carvalho, D.D. Epigenetic therapy in immune-oncology. Nat Rev Cancer 19, 151-161 (2019).

24. Kim, Y., et al. PKR Senses Nuclear and Mitochondrial Signals by Interacting with Endogenous Double-Stranded RNAs. Mol Cell 71, 1051-1063 e1056 (2018). 
25. Canadas, I., et al. Tumor innate immunity primed by specific interferon-stimulated endogenous retroviruses. Nat Med 24, 1143-1150 (2018).

26. Roulois, D., et al. DNA-Demethylating Agents Target Colorectal Cancer Cells by Inducing Viral Mimicry by Endogenous Transcripts. Cell 162, 961-973 (2015).

27. Stone, M.L., et al. Epigenetic therapy activates type I interferon signaling in murine ovarian cancer to reduce immunosuppression and tumor burden. Proc Natl Acad Sci U S A 114, E10981-E10990 (2017).

28. Eisenberg, E. \& Levanon, E.Y. A-to-I RNA editing - immune protector and transcriptome diversifier. Nat Rev Genet 19, 473-490 (2018).

29. Hartner, J.C., Walkley, C.R., Lu, J. \& Orkin, S.H. ADAR1 is essential for the maintenance of hematopoiesis and suppression of interferon signaling. Nat Immunol 10, 109-115 (2009).

30. Mannion, N.M., et al. The RNA-editing enzyme ADAR1 controls innate immune responses to RNA. Cell Rep 9, 1482-1494 (2014).

31. Linder, P. \& Jankowsky, E. From unwinding to clamping - the DEAD box RNA helicase family. Nat Rev Mol Cell Biol 12, 505-516 (2011).

32. Bourgeois, C.F., Mortreux, F. \& Auboeuf, D. The multiple functions of RNA helicases as drivers and regulators of gene expression. Nat Rev Mol Cell Biol 17, 426-438 (2016).

33. Ariumi, Y. Multiple functions of DDX3 RNA helicase in gene regulation, tumorigenesis, and viral infection. Front Genet 5, 423 (2014).

34. Song, H. \& Ji, X. The mechanism of RNA duplex recognition and unwinding by DEADbox helicase DDX3X. Nat Commun 10, 3085 (2019).

35. Linder, P. \& Fuller-Pace, F.V. Looking back on the birth of DEAD-box RNA helicases. Biochim Biophys Acta 1829, 750-755 (2013).

36. Shih, J.W. \& Lee, Y.H. Human DExD/H RNA helicases: emerging roles in stress survival regulation. Clin Chim Acta 436, 45-58 (2014).

37. Bol, G.M., Xie, M. \& Raman, V. DDX3, a potential target for cancer treatment. Mol Cancer 14, 188 (2015).

38. Fuller-Pace, F.V. DEAD box RNA helicase functions in cancer. RNA Biol 10, 121-132 (2013).

39. Samal, S.K., Routray, S., Veeramachaneni, G.K., Dash, R. \& Botlagunta, M. Ketorolac salt is a newly discovered DDX3 inhibitor to treat oral cancer. Sci Rep 5, 9982 (2015).

40. Xie, M., et al. NZ51, a ring-expanded nucleoside analog, inhibits motility and viability of breast cancer cells by targeting the RNA helicase DDX3. Oncotarget 6, 29901-29913 (2015).

41. Heerma van Voss, M.R., et al. Targeting mitochondrial translation by inhibiting DDX3: a novel radiosensitization strategy for cancer treatment. Oncogene 37, 63-74 (2018).

42. Heerma van Voss, M.R., et al. Identification of the DEAD box RNA helicase DDX3 as a therapeutic target in colorectal cancer. Oncotarget 6, 28312-28326 (2015).

43. Kukhanova, M.K., Karpenko, I.L. \& Ivanov, A.V. DEAD-box RNA Helicase DDX3: Functional Properties and Development of DDX3 Inhibitors as Antiviral and Anticancer Drugs. Molecules 25(2020).

44. Heerma van Voss, M.R., et al. Combination treatment using DDX3 and PARP inhibitors induces synthetic lethality in BRCA1-proficient breast cancer. Med Oncol 34, 33 (2017).

45. Botlagunta, M., et al. Oncogenic role of DDX3 in breast cancer biogenesis. Oncogene 27, 3912-3922 (2008).

46. Heerma van Voss, M.R., et al. The prognostic effect of DDX3 upregulation in distant breast cancer metastases. Clin Exp Metastasis 34, 85-92 (2017).

47. Lukacs, N. Detection of virus infection in plants and differentiation between coexisting viruses by monoclonal antibodies to double-stranded RNA. J Virol Methods 47, 255-272 (1994). 
48. Schonborn, J., et al. Monoclonal antibodies to double-stranded RNA as probes of RNA structure in crude nucleic acid extracts. Nucleic Acids Res 19, 2993-3000 (1991).

49. Weber, F., Wagner, V., Rasmussen, S.B., Hartmann, R. \& Paludan, S.R. Doublestranded RNA is produced by positive-strand RNA viruses and DNA viruses but not in detectable amounts by negative-strand RNA viruses. J Virol 80, 5059-5064 (2006).

50. Liu, M., et al. Vitamin C increases viral mimicry induced by 5-aza-2'-deoxycytidine. Proc Natl Acad Sci U S A 113, 10238-10244 (2016).

51. Ferreira, C.B., et al. Lentiviral Vector Production Titer Is Not Limited in HEK293T by Induced Intracellular Innate Immunity. Mol Ther Methods Clin Dev 17, 209-219 (2020).

52. Woo, S.R., et al. STING-dependent cytosolic DNA sensing mediates innate immune recognition of immunogenic tumors. Immunity 41, 830-842 (2014).

53. Frohlich, A., et al. DEAD-box RNA helicase DDX3 connects CRM1-dependent nuclear export and translation of the HIV-1 unspliced mRNA through its N-terminal domain. Biochim Biophys Acta 1859, 719-730 (2016).

54. Lai, M.C., Lee, Y.H. \& Tarn, W.Y. The DEAD-box RNA helicase DDX3 associates with export messenger ribonucleoproteins as well as tip-associated protein and participates in translational control. Mol Biol Cell 19, 3847-3858 (2008).

55. Kudo, N., et al. Leptomycin B inhibition of signal-mediated nuclear export by direct binding to CRM1. Exp Cell Res 242, 540-547 (1998).

56. Bass, B.L. RNA editing by adenosine deaminases that act on RNA. Annu Rev Biochem 71, 817-846 (2002).

57. Fritzell, K., Xu, L.D., Otrocka, M., Andreasson, C. \& Ohman, M. Sensitive ADAR editing reporter in cancer cells enables high-throughput screening of small molecule libraries. Nucleic Acids Res 47, e22 (2019).

58. Liu, H., et al. Tumor-derived IFN triggers chronic pathway agonism and sensitivity to ADAR loss. Nat Med 25, 95-102 (2019).

59. Pujantell, M., et al. RNA editing by ADAR1 regulates innate and antiviral immune functions in primary macrophages. Sci Rep 7, 13339 (2017).

60. McDonald, E.R., 3rd, et al. Project DRIVE: A Compendium of Cancer Dependencies and Synthetic Lethal Relationships Uncovered by Large-Scale, Deep RNAi Screening. Cell 170, 577-592 e510 (2017).

61. McFarland, J.M., et al. Improved estimation of cancer dependencies from large-scale RNAi screens using model-based normalization and data integration. Nat Commun $\mathbf{9}$, 4610 (2018).

62. Blango, M.G. \& Bass, B.L. Identification of the long, edited dsRNAome of LPSstimulated immune cells. Genome Res 26, 852-862 (2016).

63. Ruggieri, A. \& Stoecklin, G. A signal to condense. Nat Chem Biol 15, 5-6 (2019).

64. Boeynaems, S., et al. Protein Phase Separation: A New Phase in Cell Biology. Trends Cell Biol 28, 420-435 (2018).

65. Saito, M., et al. Acetylation of intrinsically disordered regions regulates phase separation. Nat Chem Biol 15, 51-61 (2019).

66. Kim, Y. \& Myong, S. RNA Remodeling Activity of DEAD Box Proteins Tuned by Protein Concentration, RNA Length, and ATP. Mol Cell 63, 865-876 (2016).

67. Tian, B., Bevilacqua, P.C., Diegelman-Parente, A. \& Mathews, M.B. The doublestranded-RNA-binding motif: interference and much more. Nat Rev Mol Cell Biol 5, 1013-1023 (2004).

68. Oshiumi, H., Sakai, K., Matsumoto, M. \& Seya, T. DEAD/H BOX 3 (DDX3) helicase binds the RIG-I adaptor IPS-1 to up-regulate IFN-beta-inducing potential. Eur J Immunol 40, 940-948 (2010).

69. Schroder, M., Baran, M. \& Bowie, A.G. Viral targeting of DEAD box protein 3 reveals its role in TBK1/IKKepsilon-mediated IRF activation. EMBO J 27, 2147-2157 (2008). 
70. Soulat, D., et al. The DEAD-box helicase DDX3X is a critical component of the TANKbinding kinase 1-dependent innate immune response. EMBO J 27, 2135-2146 (2008).

71. Gu, L., Fullam, A., Brennan, R. \& Schroder, M. Human DEAD box helicase 3 couples IkappaB kinase epsilon to interferon regulatory factor 3 activation. $\mathrm{Mol} \mathrm{Cell} \mathrm{Biol} \mathbf{3 3}$, 2004-2015 (2013).

72. Fullam, A. \& Schroder, M. DExD/H-box RNA helicases as mediators of anti-viral innate immunity and essential host factors for viral replication. Biochim Biophys Acta 1829, 854-865 (2013).

73. Li, Q., Pene, V., Krishnamurthy, S., Cha, H. \& Liang, T.J. Hepatitis C virus infection activates an innate pathway involving IKK-alpha in lipogenesis and viral assembly. Nat Med 19, 722-729 (2013).

74. Gringhuis, S.I., et al. HIV-1 blocks the signaling adaptor MAVS to evade antiviral host defense after sensing of abortive HIV-1 RNA by the host helicase DDX3. Nat Immunol 18, 225-235 (2017).

75. Gu, L., Fullam, A., McCormack, N., Hohn, Y. \& Schroder, M. DDX3 directly regulates TRAF3 ubiquitination and acts as a scaffold to co-ordinate assembly of signalling complexes downstream from MAVS. Biochem J 474, 571-587 (2017).

76. Szappanos, D., et al. The RNA helicase DDX3X is an essential mediator of innate antimicrobial immunity. PLoS Pathog 14, e1007397 (2018).

77. Samir, P., et al. DDX3X acts as a live-or-die checkpoint in stressed cells by regulating NLRP3 inflammasome. Nature 573, 590-594 (2019).

78. Gannon, H.S., et al. Identification of ADAR1 adenosine deaminase dependency in a subset of cancer cells. Nat Commun 9, 5450 (2018).

79. Liddicoat, B.J., et al. RNA editing by ADAR1 prevents MDA5 sensing of endogenous dsRNA as nonself. Science 349, 1115-1120 (2015).

80. Lamers, M.M., van den Hoogen, B.G. \& Haagmans, B.L. ADAR1: "Editor-in-Chief" of Cytoplasmic Innate Immunity. Front Immunol 10, 1763 (2019).

81. Borden, E.C. Interferons alpha and beta in cancer: therapeutic opportunities from new insights. Nat Rev Drug Discov 18, 219-234 (2019).

82. Iurescia, S., Fioretti, D. \& Rinaldi, M. Targeting Cytosolic Nucleic Acid-Sensing Pathways for Cancer Immunotherapies. Front Immunol 9, 711 (2018).

83. Topper, M.J., et al. Epigenetic Therapy Ties MYC Depletion to Reversing Immune Evasion and Treating Lung Cancer. Cell 171, 1284-1300 e1221 (2017).

84. Fu, C. \& Jiang, A. Dendritic Cells and CD8 T Cell Immunity in Tumor Microenvironment. Front Immunol 9, 3059 (2018).

85. Han, C., et al. Amplification of USP13 drives ovarian cancer metabolism. Nat Commun 7, 13525 (2016).

86. Ritchie, M.E., et al. limma powers differential expression analyses for RNA-sequencing and microarray studies. Nucleic Acids Res 43, e47 (2015).

87. Sergushichev, A.A. An algorithm for fast preranked gene set enrichment analysis using cumulative statistic calculation. bioRxiv, 060012 (2016).

88. Yu, G., Wang, L.G., Han, Y. \& He, Q.Y. clusterProfiler: an R package for comparing biological themes among gene clusters. OMICS 16, 284-287 (2012).

89. Breuer, K., et al. InnateDB: systems biology of innate immunity and beyond--recent updates and continuing curation. Nucleic Acids Res 41, D1228-1233 (2013). 
Fig. 1: Loss of DDX3X increases the expression of genes in antiviral innate immune response in breast cancer cells.

a, Genome-wide transcriptome analysis in DDX3X-control (shNS) or -knockdown (shDDX3X) MCF7 cells using a next generation RNA deep sequencing. DEGs (FDR 0.05 , fold change $>1.5$ or <-1.5) in shNS vs. shDDX3X are shown in a MA-plot (labeled in red) and in a heat map.

b, IPA analysis shows top canonical pathways of DEGs in DDX3X-control or -knockdown (KD) MCF7.

c, GSEA analysis of DEGs in DDX3X-control or -KD MCF7.

d, qRT-PCR of mRNA expression of ISGs and APP in DDX3X-control or -KD MCF7 cells.

e, qRT-PCR of ISGs expression in DDX1-KD or DDX5-KD MCF7 cells, respectively.

Data in $D$ and $E$ are representative of three independent experiments and presented as mean \pm SEM. Statistics were calculated using unpaired t-tests. ${ }^{*} P<0.05$; ${ }^{* *} P<0.01$; ${ }^{* \star \star} P<0.001$; ns, not significant.

Fig. 2: Loss of DDX3X activates type I IFN production, suppresses cell proliferation, and induces antigen presentation in cancer cells.

a, ELISA of IFN- $\alpha,-\beta$, and $-\gamma$ in the culture supernatants from DDX3X-control or -depleted MCF7, MB453, and E0771 cells, respectively.

b. Western blot analysis of STAT1 and phosphorylated STAT1 in the DDX3X-control or depleted MCF7 and MDA-MB-453 cells, respectively.

c, Colony formation assay in DDX3X-control or -KD MCF7 cells shows cell growth inhibition by DDX3X depletion.

d, qRT-PCR of IFIT3 and OAS1 in DDX3X-control or -KD MCF7 cells after IFN- $\alpha$ treatment for 5 hours.

e, Representative flow histograms (left) and a relative mean fluorescence intensity (MFI) quantification bar graph (right) of HLA-ABC expression in the DDX3X-KD MCF7 or DDX3X-KD MDA-MB-453 cells, respectively.

f, Representative histograms (left) and a relative MFI quantification bar graph (right) of SIINFEKL bound to $\mathrm{H}-2 \mathrm{~Kb}$ expression on DDX3X deficient B16-OVA cells. Cells were treated $100 \mathrm{ng} / \mathrm{ml}$ of IFN $\gamma$ for 48 hours.

Data are representative of three independent experiments. Data are shown as mean \pm SEM of three independent experiments. Statistics were calculated using unpaired t-tests. ${ }^{*} P 0.05$; ${ }^{* *} P$ $<0.01 ;{ }^{* * *} P<0.001 ;{ }^{* * *} P<0.0001$; ns, not significant. 
Fig. 3: DDX3X inhibition triggers endogenous dsRNAs accumulation.

a-d, Endogenous dsRNA accumulation in DDX3X-control or -KD MCF7 cells analyzed by immunofluorescence (a), flow cytometry (b), northwestern blot (c), and dot blot (d) with antidsRNA specific (J2) antibody. Scale bars, $25 \mu \mathrm{m}$.

e, dsRNA accumulation in DDX3X-depleted MDA-MB-453, A375, 4T1, and B16F10 cells assessed by dot blot.

f, qRT-PCR of ERV genes in DDX3X-depleted MCF7 cells.

g, TASA-TD PCR amplified sense and antisense transcripts of the Syncytin-1 and ERV9-1 genes. A $\beta$-Actin used for a sense transcript amplification. Syncytin-1 used for a positive control for a bi-directional transcript.

$\mathbf{h}, \mathrm{qRT}-\mathrm{PCR}$ of ERV genes in DDX3X-control or -KD MDA-MB-453 cells.

Data are representative of three independent experiments. Data are shown as mean $\pm \mathrm{SEM}$.

Statistics were calculated using unpaired t-tests. ${ }^{*} P<0.05 ;{ }^{* \star} P<0.01$; ${ }^{* * *} P<0.0001$.

Fig. 4: Inhibiting DDX3X activates cytoplasmic dsRNA response signaling in cancer and MEF cells.

a, Western blot analysis of dsDNA sensor and downstream target, cGAS and STING, in DDX3X-control or -depleted MCF7 or MDA-MB-453 cells.

b, Western blot analysis of dsRNA sensors and downstream target, TLR3, PKR and elF2a, in DDX3X-control or -depleted MCF7 or -MDA-MB-453 cells.

c, Western blot analysis of dsRNA sensors, RIG-I, MDA5, and OAS1, in DDX3X-control or depleted MCF7 or MDA-MB-453 cells.

d, Western blot analysis of phosphorylation of transcription factors, NF $\kappa B$, IRF3, and IRF7, in DDX3X-control or -depleted MCF7 or MDA-MB-453 cells.

e, qRT-PCR of IFNB1 in DDX3X-control or -KD MCF7 following by MDA5 knockout.

f, Western blot analysis of phosphorylated and total NFKB, STAT1, and STAT2 in DDX3X wildtype and DDX $3 X^{\mathrm{f} / \mathrm{f}} \mathrm{MEF}$ cells after Ad-Cre virus treatment.

g, Western blot analysis of STAT1 and STAT2 in shRNA-mediated DDX3X KD MEF cells.

$\mathbf{h}$, qRT-PCR of IFNB1 in DDX3X ${ }^{\mathrm{ff}}$ MEF cells 4 days after Ad-Cre virus treatment.

i, Schema of the dsRNA-mediated type I IFN signaling in DDX3X-depleted cancer and MEF cells.

Data are representative of three independent experiments. Data are shown as mean \pm SEM. Statistics were calculated using unpaired t-tests. ${ }^{*} P<0.05$; ${ }^{* *} P<0.01$; ns, not significant. 
Fig. 5: DDX3X interacts with cytoplasmic ADAR1 and prevents the buildup of the cytoplasmic dsRNA.

a, J2 dot blot analysis of RNA extracts from cytoplasmic or nucleus fractions of the DDX3Xcontrol or -KD MCF7 cells. Electrophoresis analysis of RNAs isolated from cytoplasmic or nucleus fractions. A circle graph shows relative dot intensity (each cells' cytoplasm + nucleus = $100 \%)$.

b, Localization of DDX3X (upper) and levels of dsRNAs (lower) in cytoplasmic or nucleus fractions after leptomycin $B(L M B ; 30 n M, 16 \mathrm{~h})$ treatment.

c, J2 Immunoprecipitation with whole MCF7 cell extracts with or without 5-AzaC treatment. MCF7 cells were fixed with $1 \%$ formaldehyde and IP was performed. dsRNA-bound proteins were analyzed using western blot with anti-DDX3X or anti-IgG (light chain) antibodies.

d, Interaction of DDX3X and ADAR. Immunoprecipitation of DDX3X from whole cell extracts or cytoplasmic fraction of MCF7 cells. IP was performed with DDX3X antibody or control IgG, respectively. Western blot analyzed using anti-ADAR1 and anti-DDX3X antibodies.

$\mathbf{e}-\mathbf{g}$, mRNA expression of dsRNA sensing genes (e), ISGs (f), and IFNB1 (g) in DDX3X and ADAR1 single, or double KD cells.

$\mathbf{h}$, Determination of ADAR1 editing as the ratio between luminescence from Nluc/FFL expressed reporter plasmid. Data was calculated a relative response ratio $(R R R)=($ experimental sample ratio-negative control ratio) / (positive control ratio-negative control ratio)).

Data are representative of three independent experiments. Data represented as mean \pm SEM. Statistics were calculated using unpaired t-tests. ${ }^{*} P<0.05 ;{ }^{* *} P<0.01 ;{ }^{* * *} P<0.001$.

Fig. 6: Low DDX3X level is related to the chronic activation of the innate antiviral immune response in the various cancer cells.

a, Flowchart detailing the steps leading to the identification of differentially expressed genes (DEGs) between DDX3X ${ }^{\text {hi }}$ and DDX3X low cancer cells in CCLE (left). DDX3X ${ }^{\text {hi }}$ (top 25\%) and DDX3X low (bottom 25\%) cell line groups based on DDX3X expression. Heatmap of 386 DEGs in DDX3X ${ }^{\text {hi }}$ vs. DDX3X ${ }^{\text {low }}$ cancer cells (right).

b, Gene set enrichment analysis (GSEA) plots for 5 top scoring hallmark gene set collection from the Molecular Signatures Database (MSigDB).

c, Violin plots showing MHC I and type I IFN transcript levels in DDX3X ${ }^{\text {hi }}$ and DDX $3 X^{\text {low }}$ group.

d, Comparison of gene dependency and expression level of ADAR1 and DDX3X in the $A D A 1 R^{\text {dep }}$ vs. ADAR $1^{\text {idp }}$ (top panel) and DDX3X ${ }^{\text {hi }}$ vs. DDX3X ${ }^{\text {low }}$ (bottom panel). Gene dependency score is obtained from DeMap portal (Genetic dependency by combined RNAi). A higher score indicates that a gene is more likely to be dependent for survival.

e, Enrichment of biological processes (GO) terms in each group of differentially expressed immune-related genes (DEIGs) between ADA1R ${ }^{\text {dep }}$ vs. ADAR $1^{\text {idp }}$ and DDX3X ${ }^{\text {hi }}$ vs. DDX3X ${ }^{\text {low }}$ groups (FDR<0.05). Bars indicate statistical significance shown as $-\log 10$ of $p$ value. Venn diagram depicting the number of DEIGs between two groups (right). 
Fig. 7: Inhibition of DDX3X suppresses breast tumor growth and enhances anti-tumor immunity.

a, Tumor growth was monitored with calipers every three days. Mice bearing 4T1 tumors expressing doxycycline-inducible DDX3X shRNA were treated with (Dox+) or without doxycycline (Dox-) water.

b, Tumor volumes and weights measured after dissection.

c, Metastatic tumor nodules in lung counted after dissection.

d, Representative dot plots showing each cell markers expression of CD45+ cells isolated from tumor. CD3+; T cell, CD20+; B cell, F4/80+; Macrophage, CD11c; dendritic cell, NK1.1; NK cell. e, Quantitative presentation of upper right quadrant from Fig. 6d.

f, Representative dot plots showing CD4+ T cells or CD8+ T cells of CD45+ cells from tumors (left). Quantitative presentation of left dot plots (CD4+ T cells in lower right quadrant, CD8+ T cells in upper left quadrant).

g, Representative dot plots showing activation profiles of CD8+ T cells of CD45+ cells isolated from tumors (left). Quantitative presentation of the upper right quadrants showing CD3+CD8+CD69+ or CD3+CD8+CD44+ T cells

h, Representative images of immunohistochemistry staining for CD8, perforin, and Ki67 in sections of tumors and quantitative data. Scare bars: $50 \mu \mathrm{m}$.

i, Quantification of CD8 + cells in immunohistochemistry.

Data are represented as mean \pm SD. $n=8$ mice per group. Unpaired t-tests. ${ }^{*} P<0.05$; ${ }^{* *} P<$ $0.01 ;{ }^{* * *} P<0.001 ;{ }^{* * * *} P<0.0001$; ns, not significant. 
a

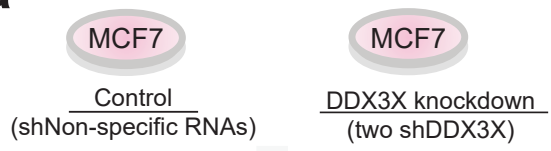

Next-generation RNA deep sequencing

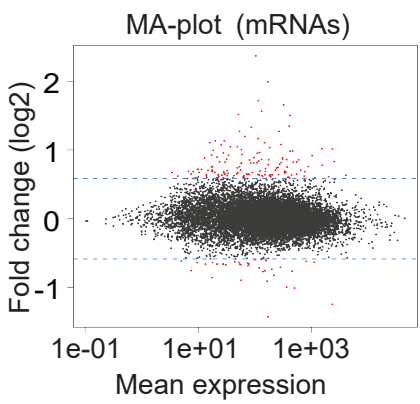

b

\begin{tabular}{lcc}
\multicolumn{1}{c}{ Top Canonical Pathways (IPA) } & p-value & Overlap \\
\hline Antigen Presentation Pathway & $1.02 \mathrm{E}-14$ & $29.7 \%(11 / 37)$ \\
Interferon Signaling & $3.72 \mathrm{E}-13$ & $27.8 \%(10 / 36)$ \\
UVA-Induced MAPK Signaling & $1.12 \mathrm{E}-05$ & $8.0 \%(7 / 88)$ \\
Pattern Recognition of Bacteria and Virus & $1.31 \mathrm{E}-05$ & $6.4 \%(8 / 125)$ \\
Retinoic acid Mediated Apoptosis Signaling & $1.42 \mathrm{E}-05$ & $9.8 \%(6 / 61)$
\end{tabular}

C

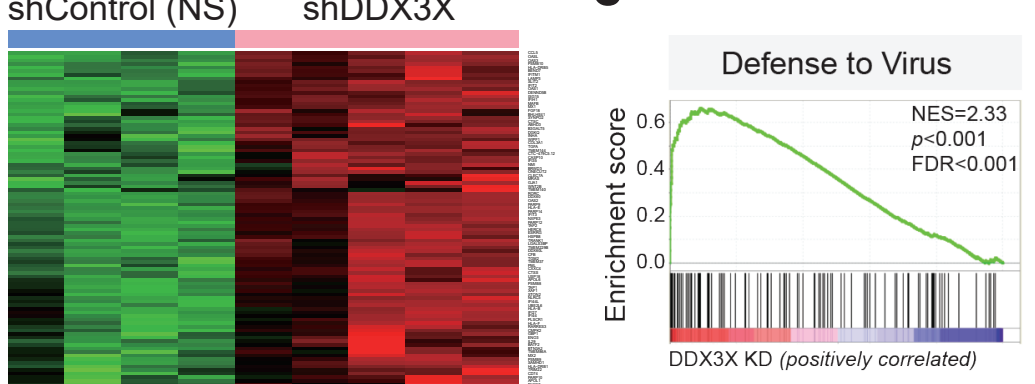

Response to Type I IFN

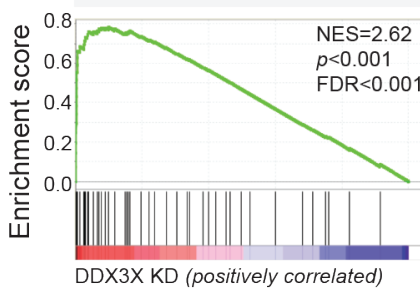

IFN alpha response

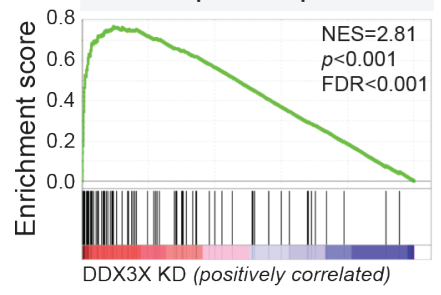

APP via MHC class I

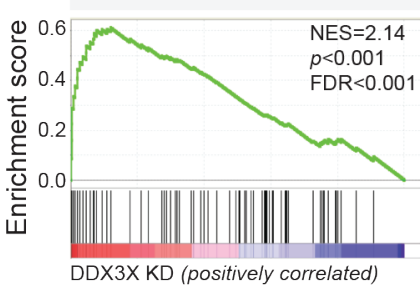

Innate immune response

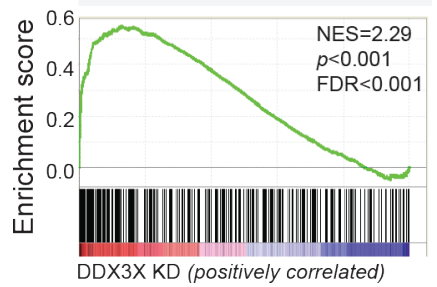

MHC protein complex

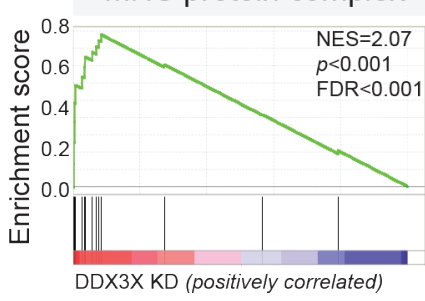

d

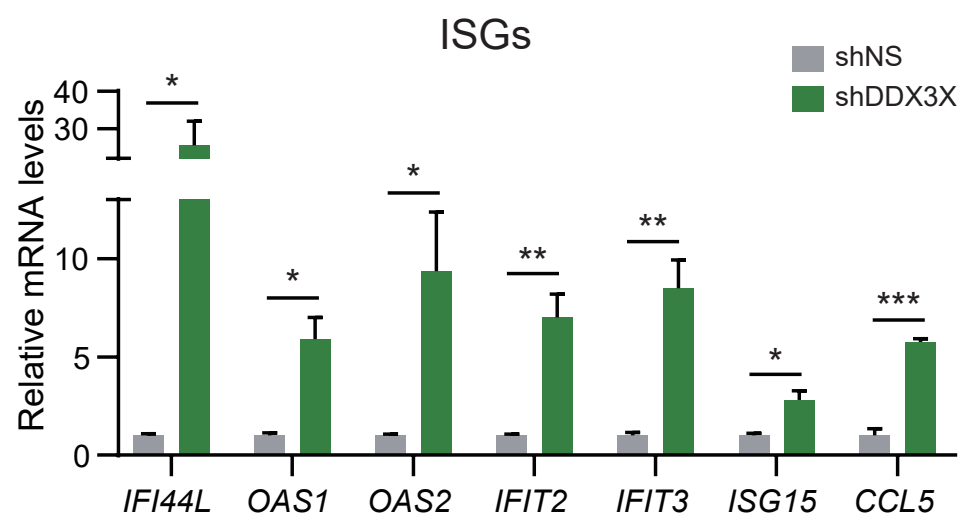

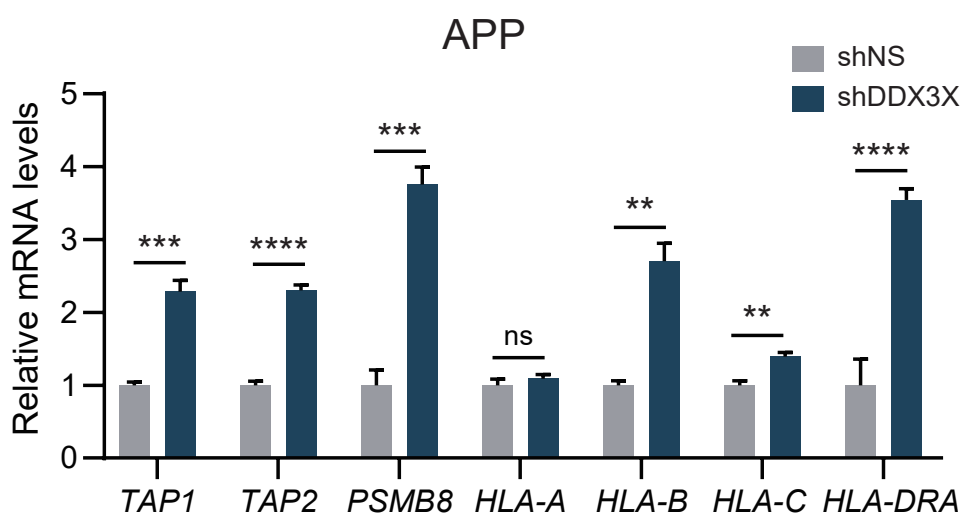

e

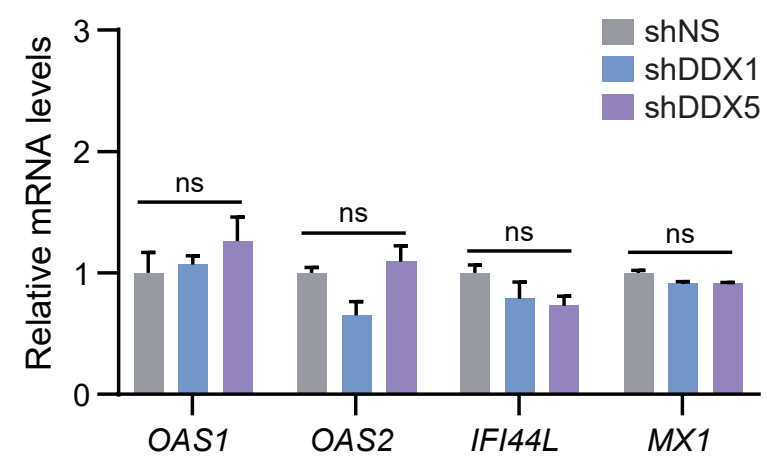


bioRxiv preprint doi: https://doi.org/10.1101/2020.09.09.289587; this version posted September 10, 2020. The copyright holder for this preprint (which was not certified by peer review) is the author/funder. All rights reserved. No reuse allowed without permission.

Fig. 2

a
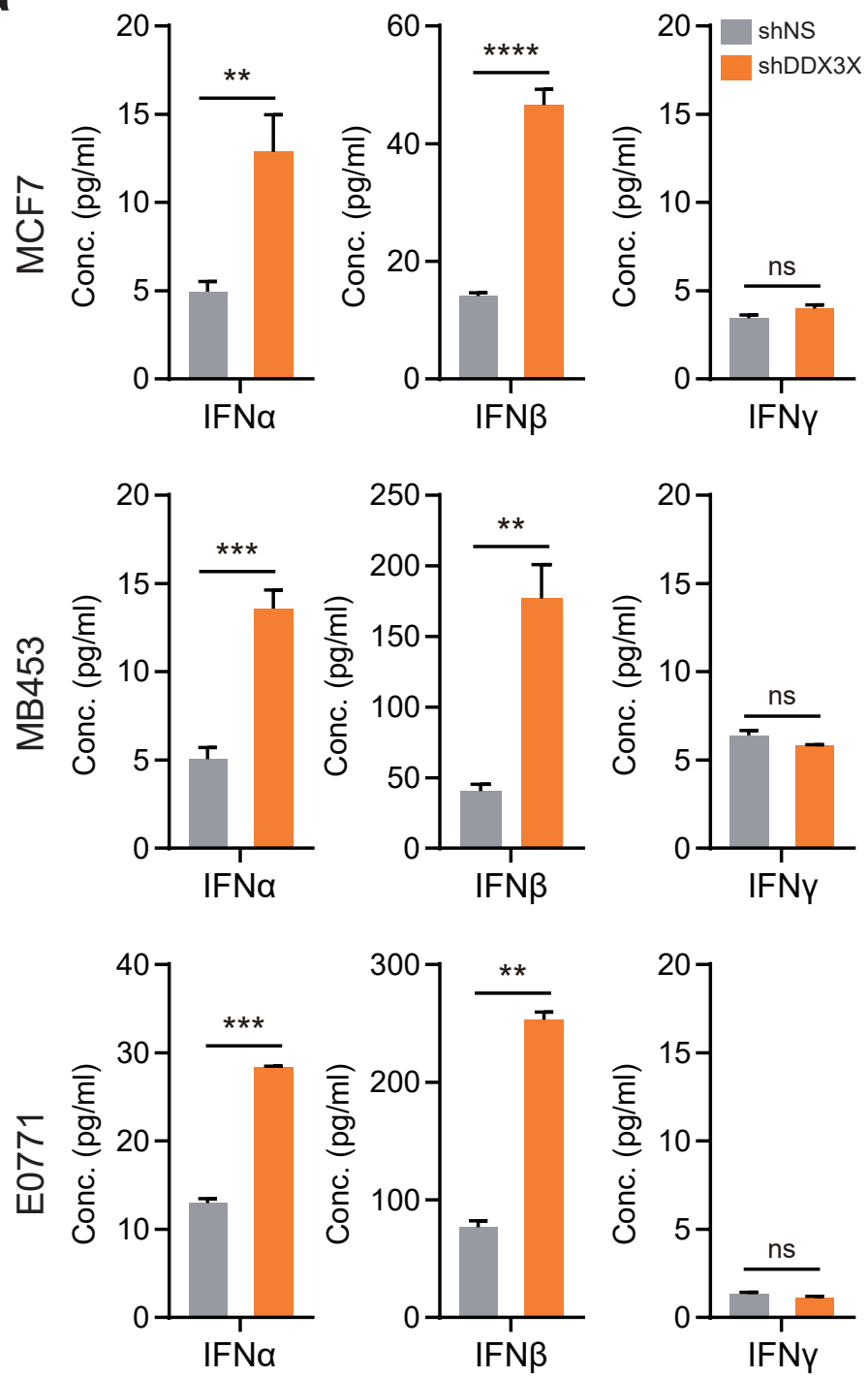
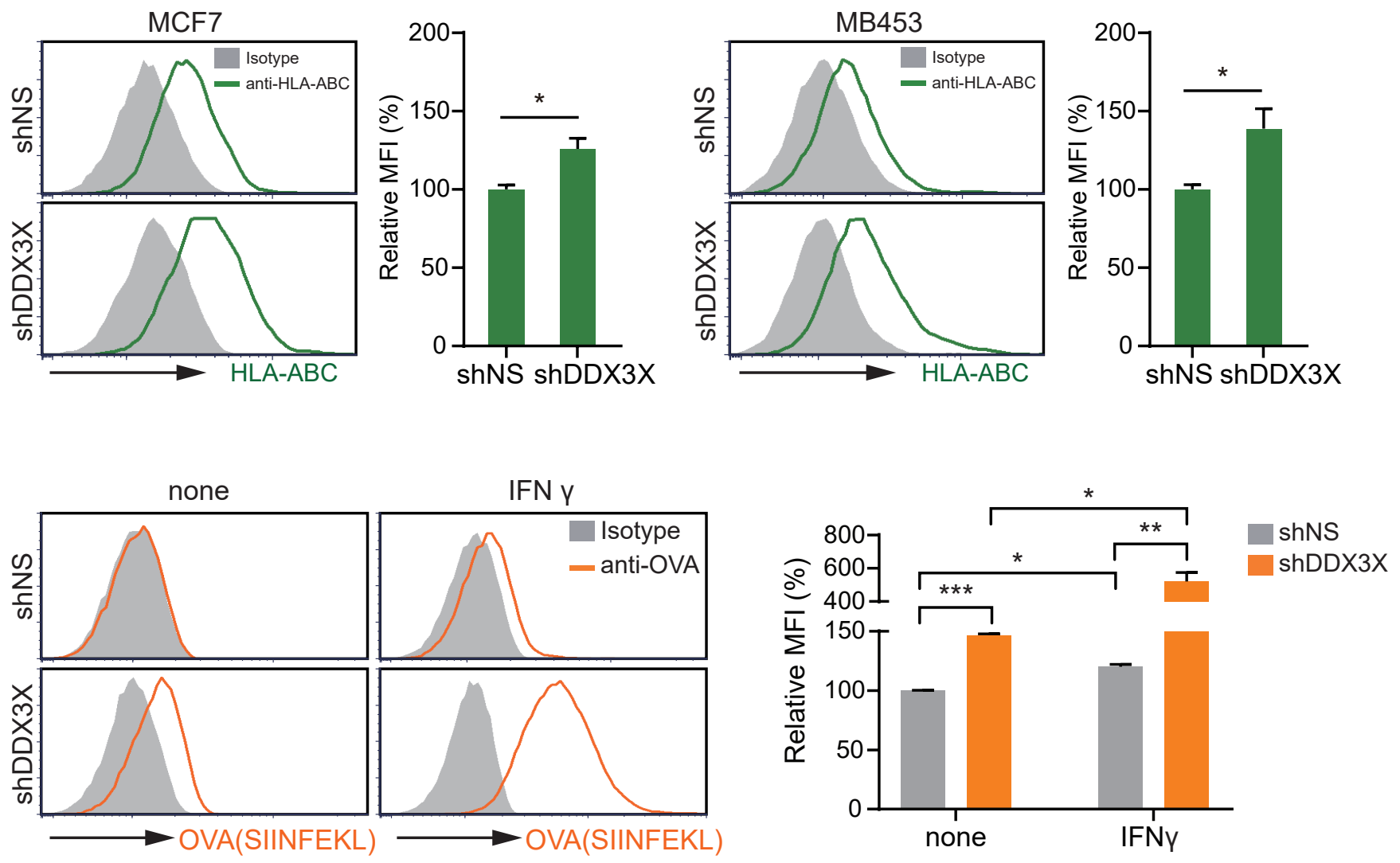

(which was not certified by peer review) is the author/funder. All rights reserved. No reuse allowed without permission.

a

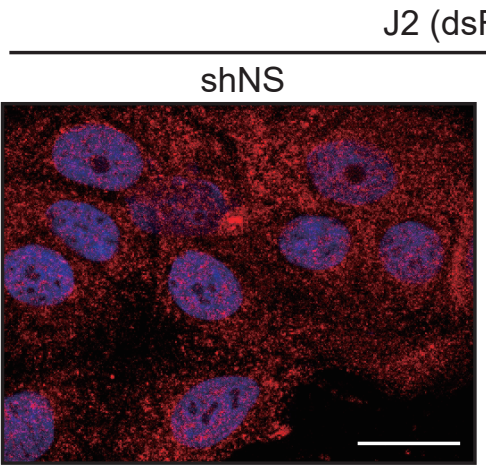

C

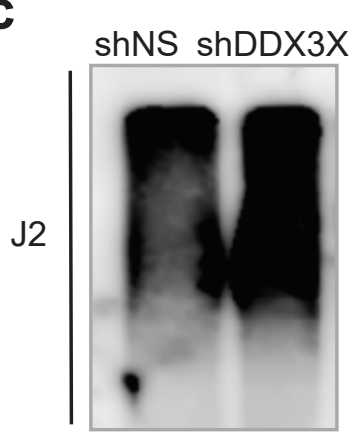

f

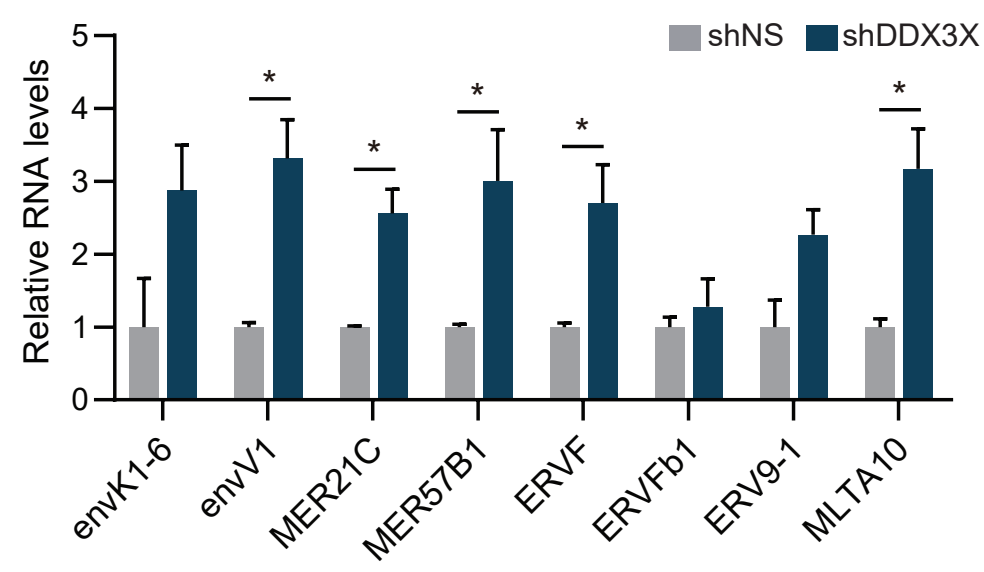

h

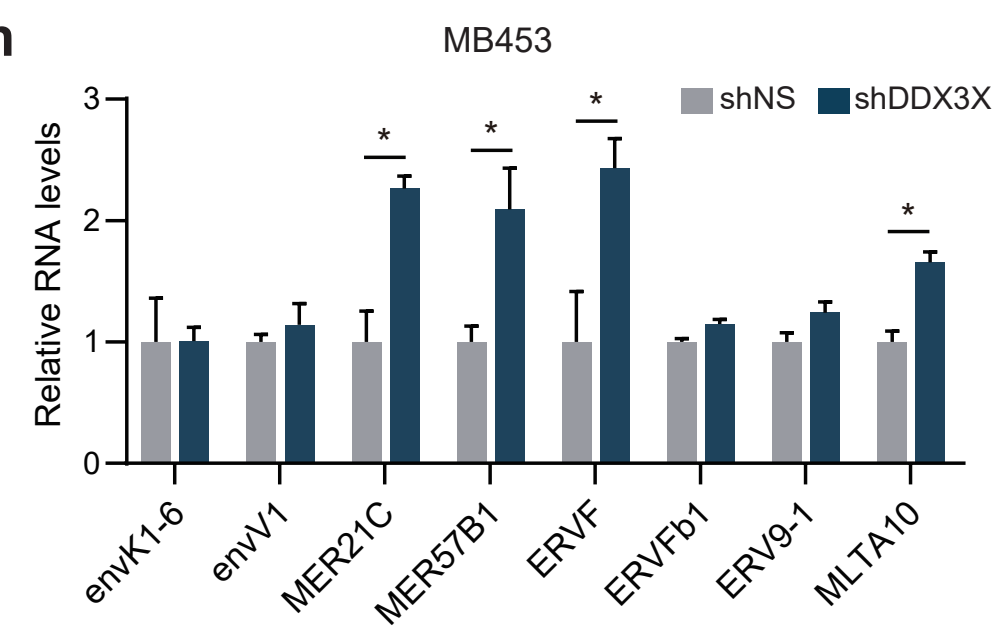

b
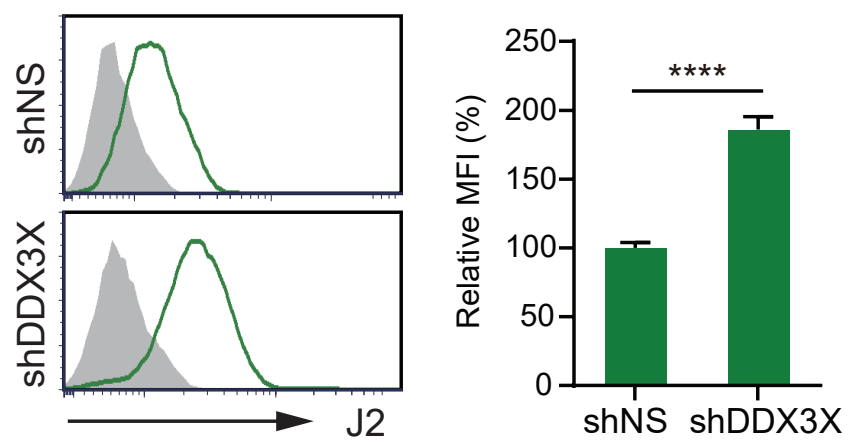

e

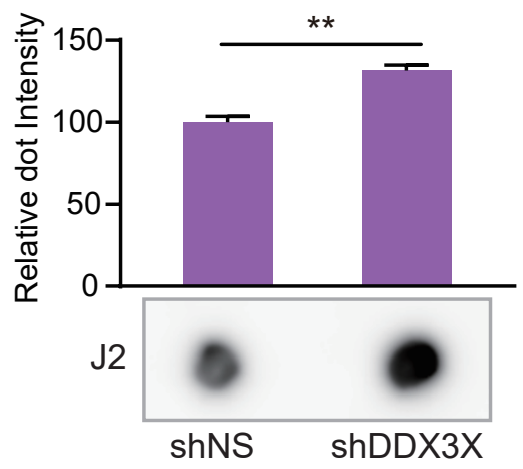

g

\begin{tabular}{rcccc} 
GS (PCR) & s & s & as & as \\
Primer TAG & + & + & + & + \\
\cline { 2 - 5 } cDNA & s & as & s & as
\end{tabular}

Syncytin-1

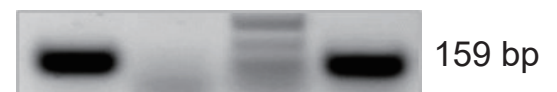

$339 \mathrm{bp}$

$487 \mathrm{bp}$
ERV9-1

$\beta$-Actin

shNS shDDX3X

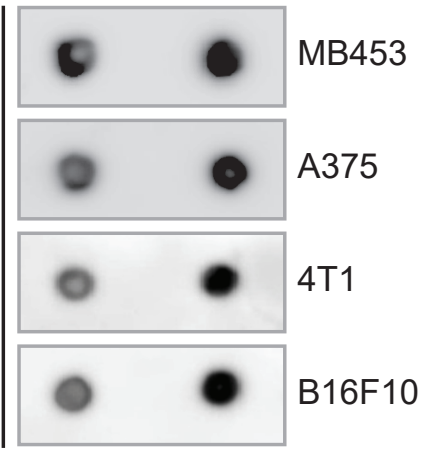

$\mathrm{J} 2$
(dsRNAs

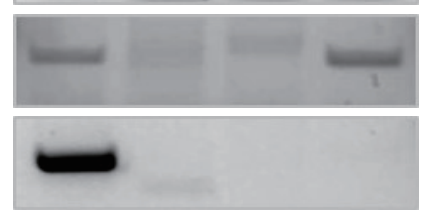

159 bp

( 
a

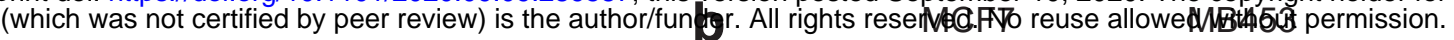

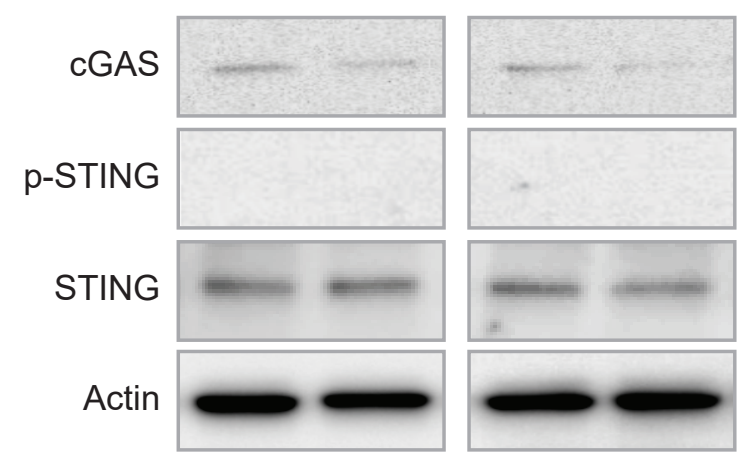

C

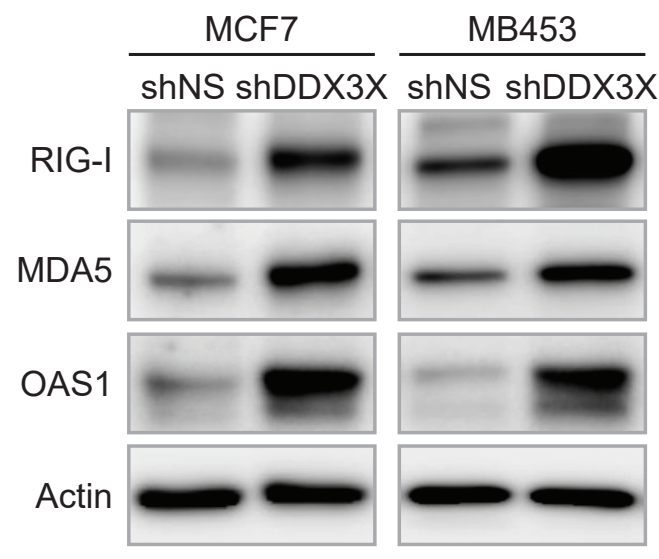

d

g

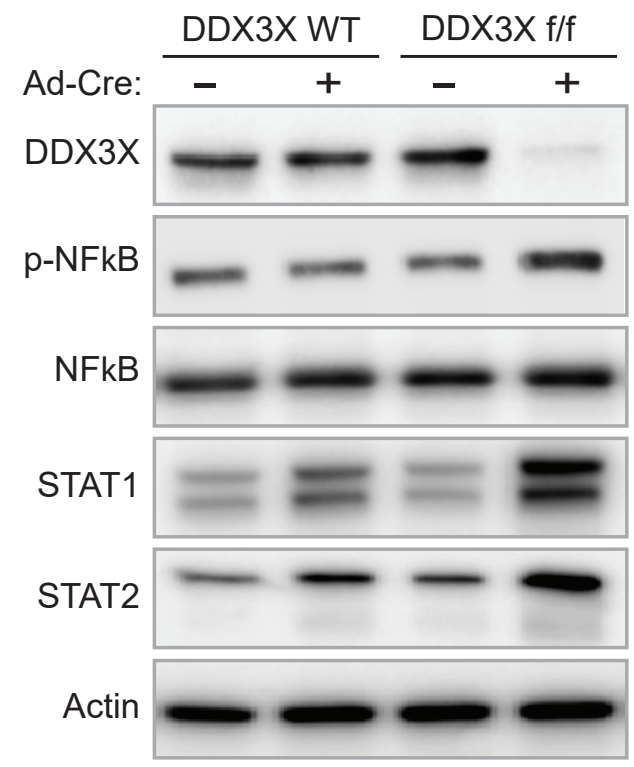

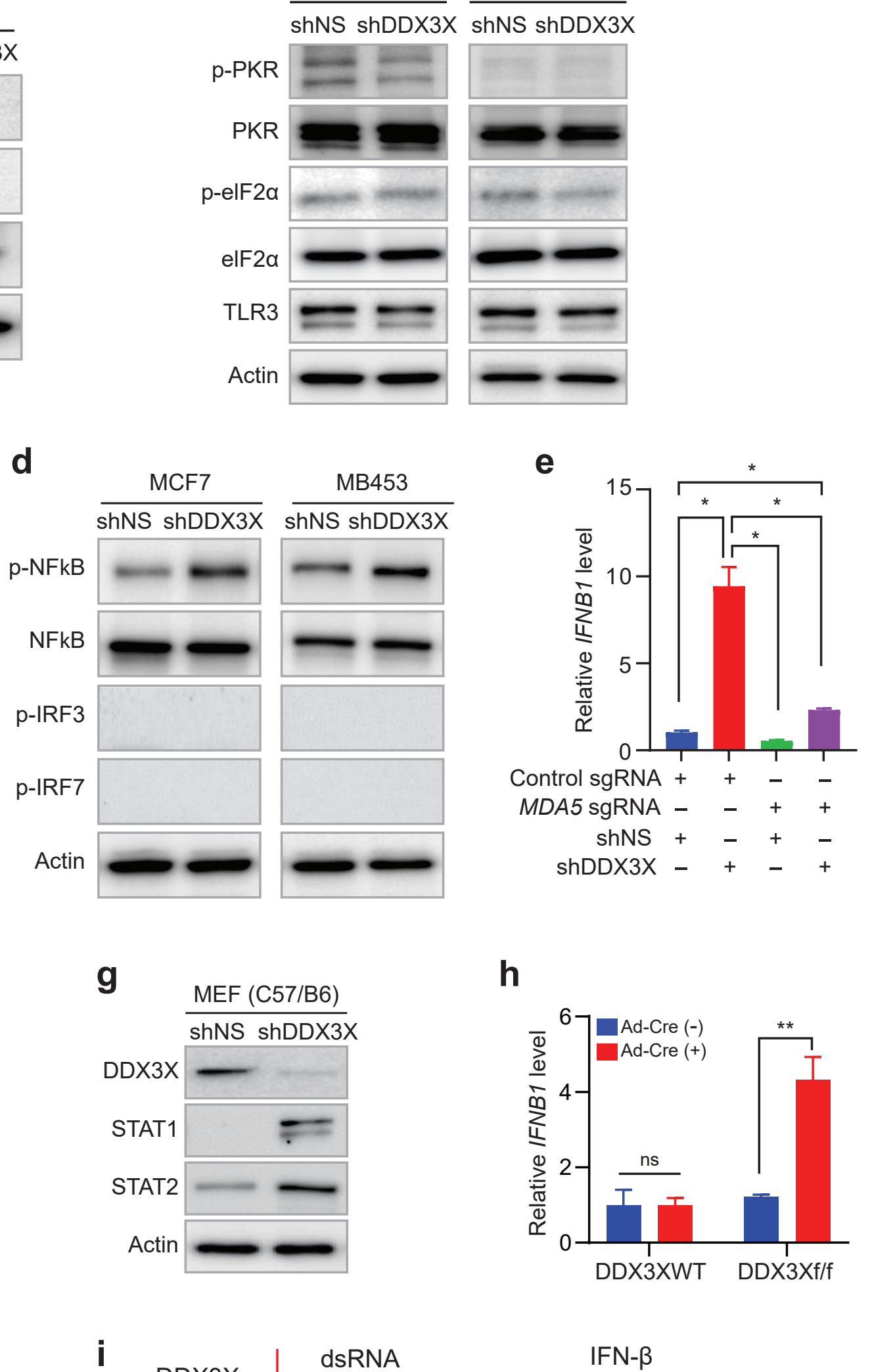
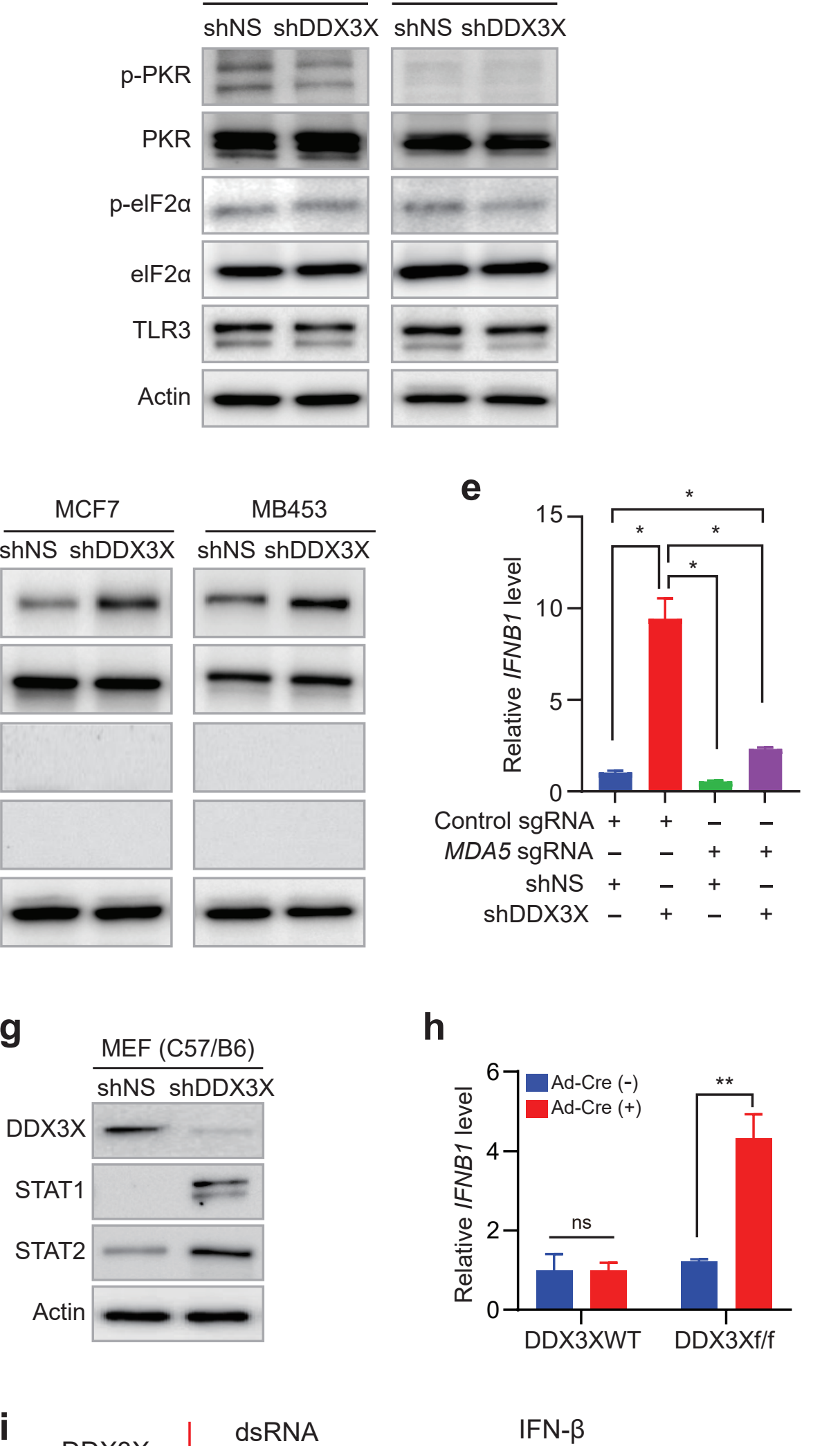

e

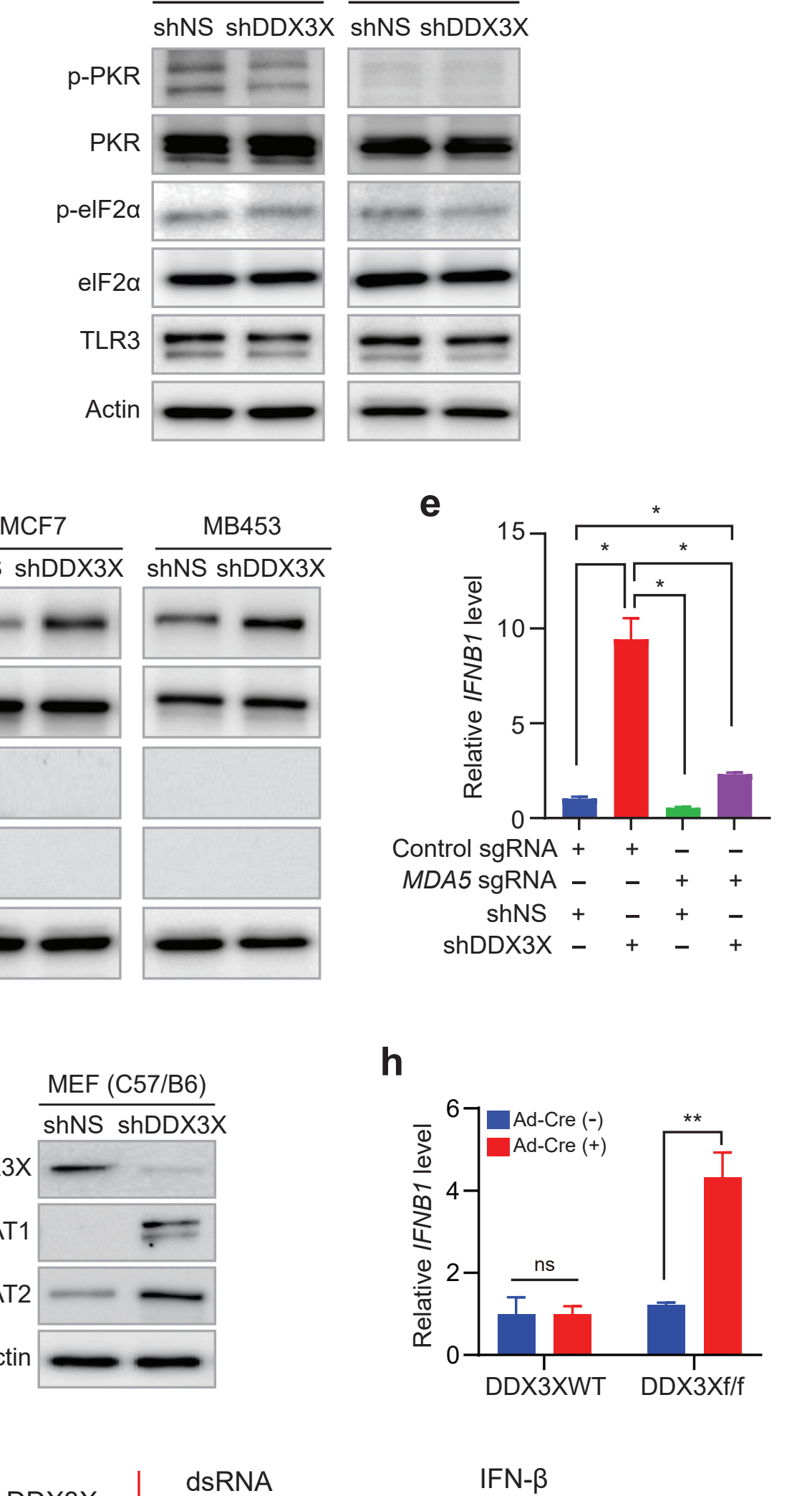

Control sgRNA ++-

MDA5 sgRNA - -++

shNS $+\quad-\quad+\quad-$

$\operatorname{shDDX} 3 \times-+\quad+$

h

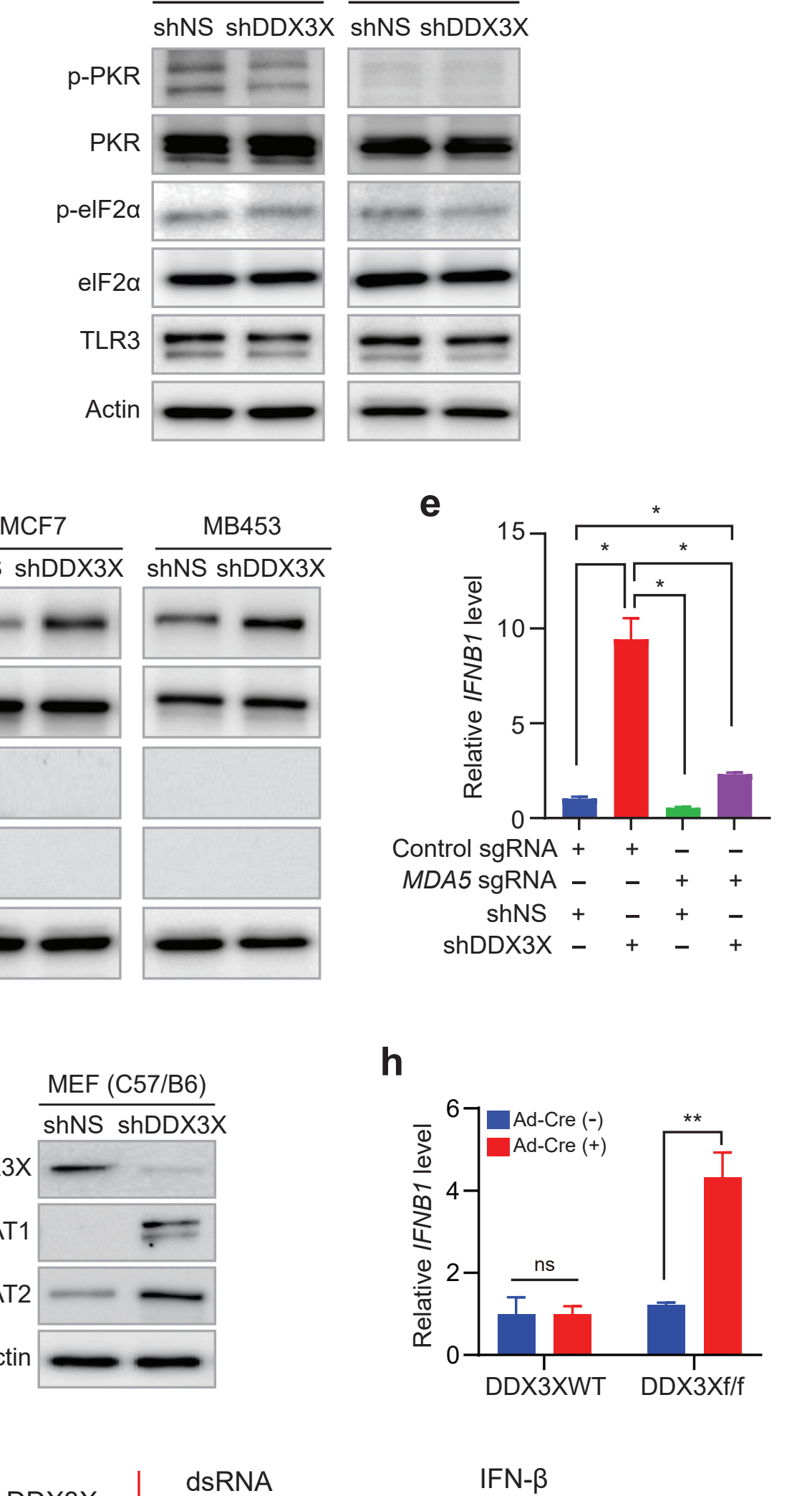

IFN- $\beta$
DDX3X $-\begin{gathered}\text { dsRNA } \\ \text { accumulation }\end{gathered}$

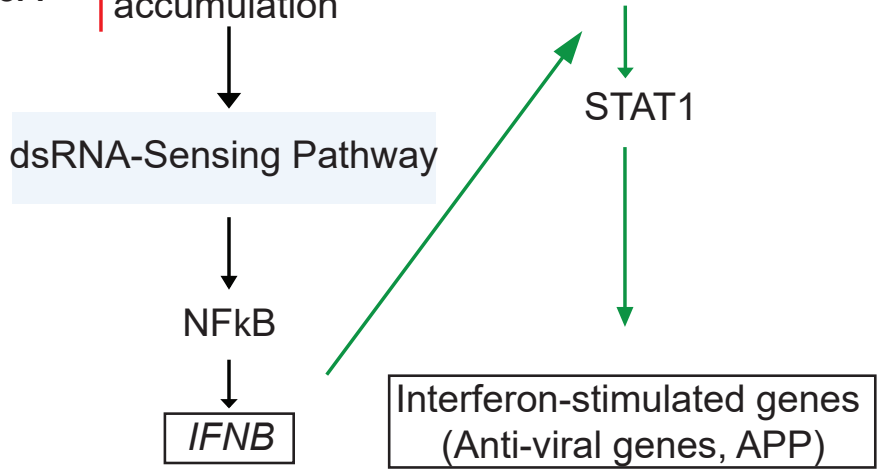



(which was not certified by peer review) is the author/funder. All rights reserved. No reuse allowed without permission.

Fig. 5

a

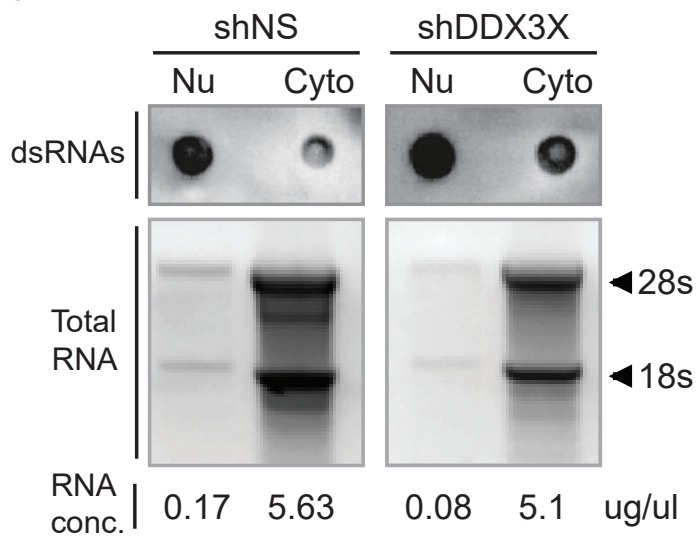

C

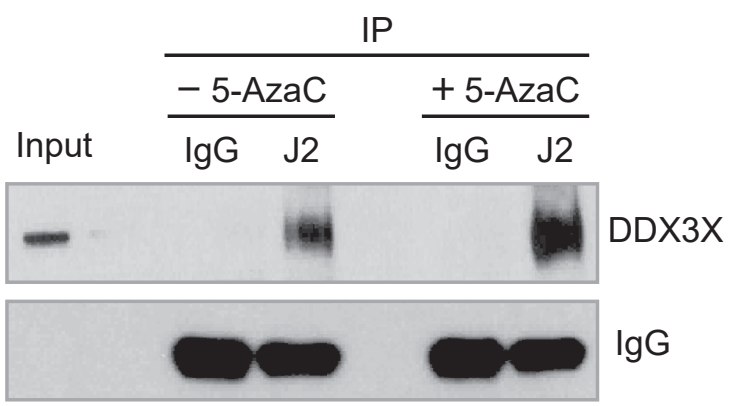

Nucleus

Cytoplasm

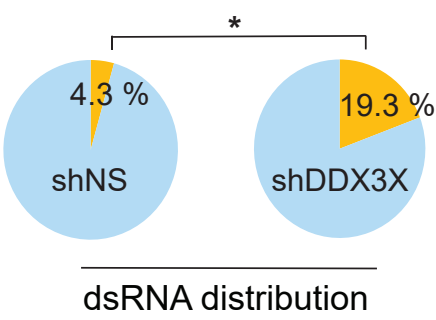

d

ADAR1 (p150)

ADAR1 (p110)

$\frac{\text { MCF7 (Whole) }}{\text { Input } \quad \operatorname{lgG} \text { DDX3X:IP }}$

$\operatorname{DDX} 3 \mathrm{X}$

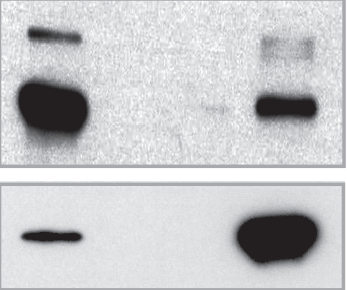

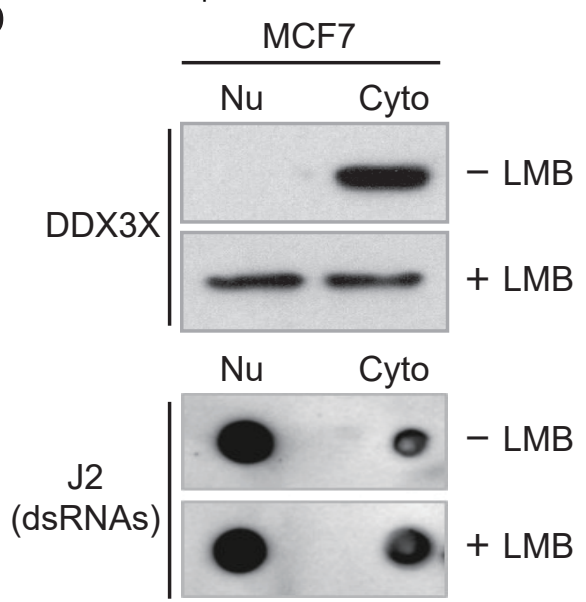

e
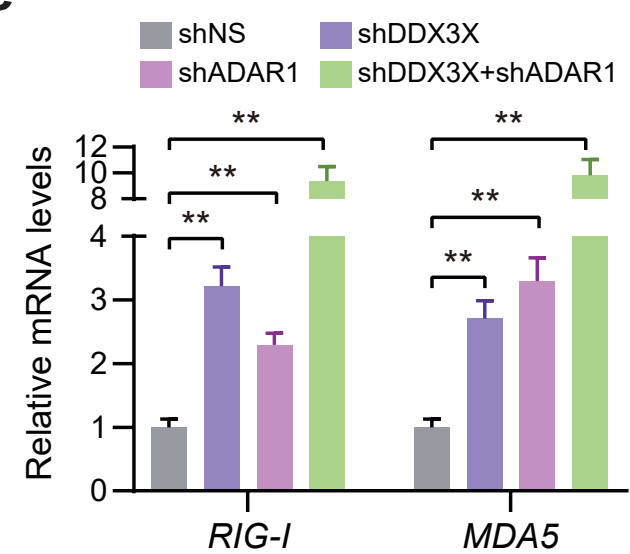

g

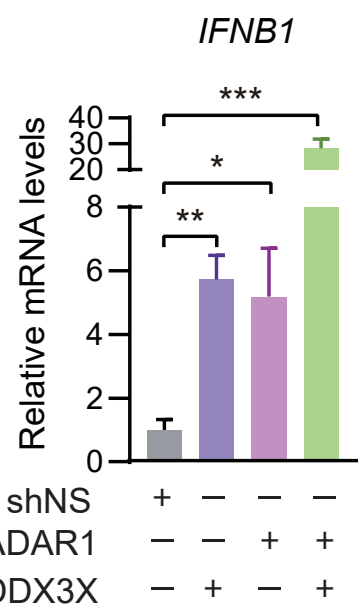

f

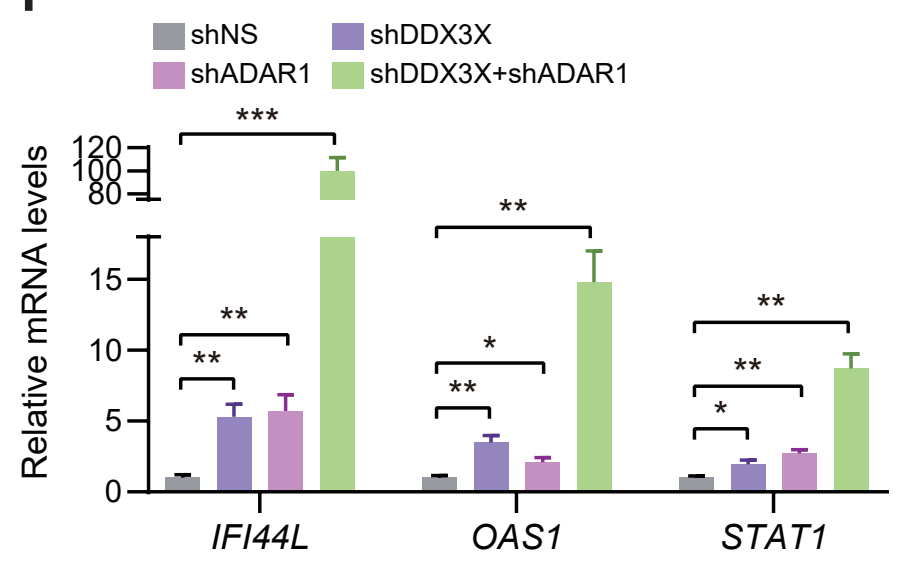

MCF7 (Cytoplasmic)

(p150)

(p110)

Input IgG DDX3X:IP

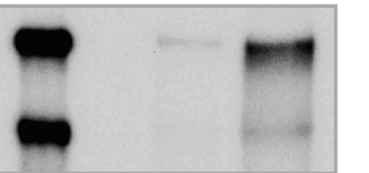

DDX3X

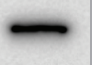

h

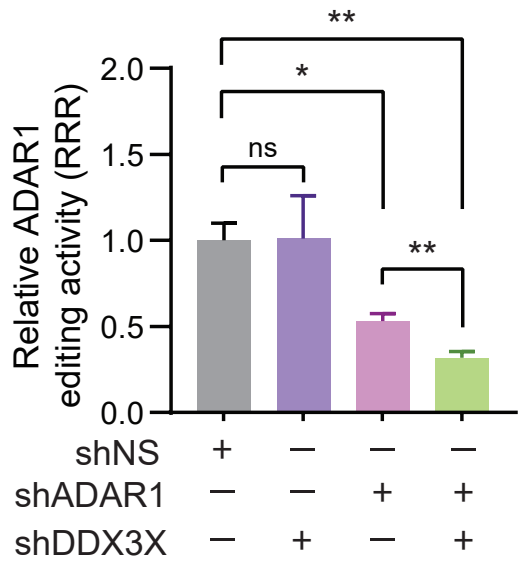


bioRxiv preprint doi: https://doi.org/10.1101/2020.09.09.289587; this version posted September 10, 2020. The copyright holder for this preprint

Fig. 6

a (which was not certified by peer review) ishthe author/funder. All rights res
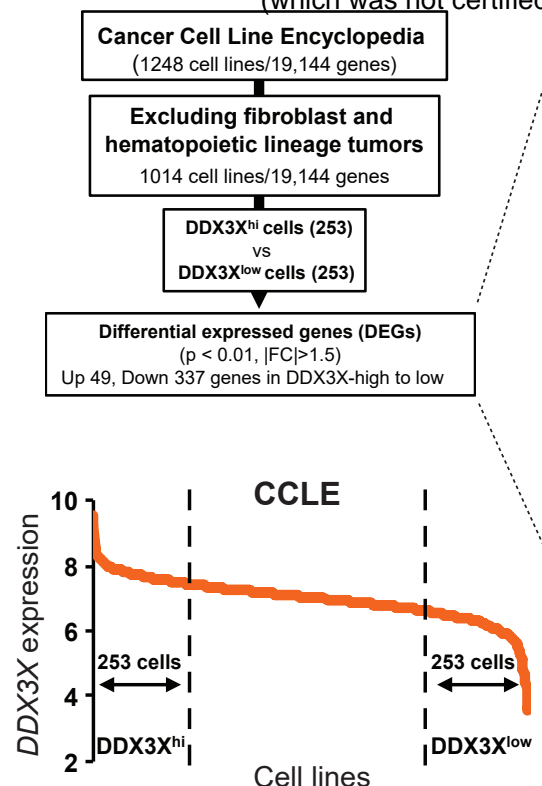

C
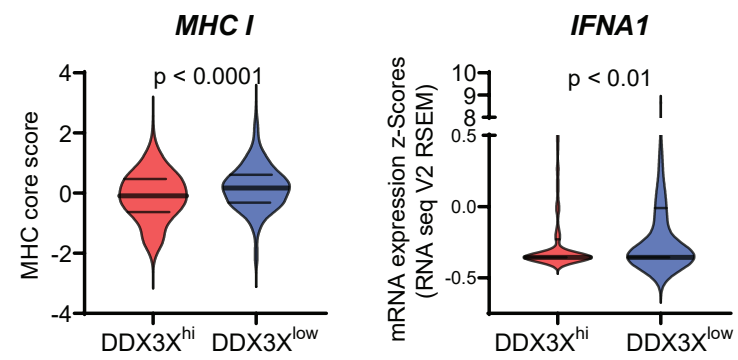

IFNB1

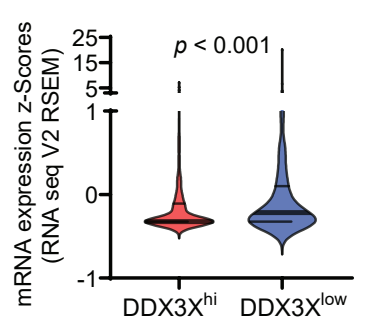

IFNE

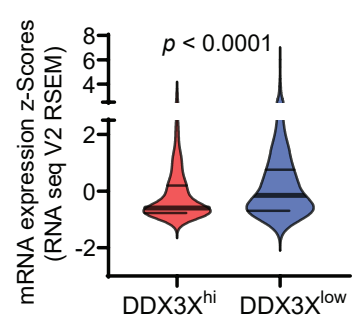

d
ADAR1-dependency

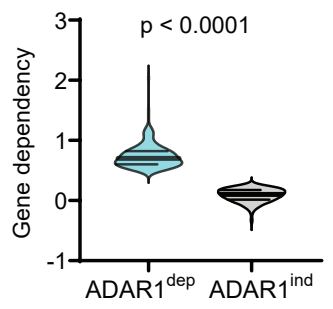

ADAR1-dependency

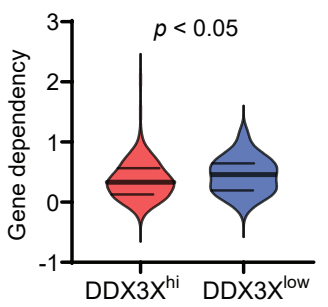

Top 5 Enriched Gene Set in DDX3X ${ }^{\text {hi }}$ vs DDX3X ${ }^{\text {low }}$

Pathway

Gene ranks NES pval

G2M-CHECKPOINT | 2.78 1.0e-01

E2F TARGETS

MITOTIC_SPINDLE

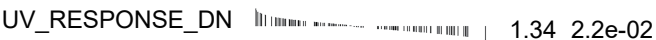

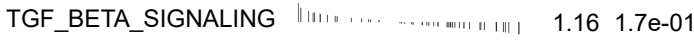

FATTY_ACID_METABOLISM "

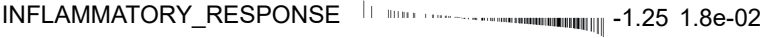

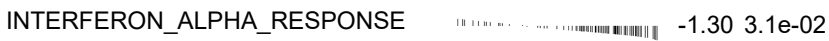

ALLOGRAFT_REJECTION I"1 "

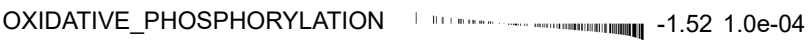

$\operatorname{DDX} 3 X^{\text {hi }} \quad$ DDX3 $x^{\text {low }}$
ADAR1

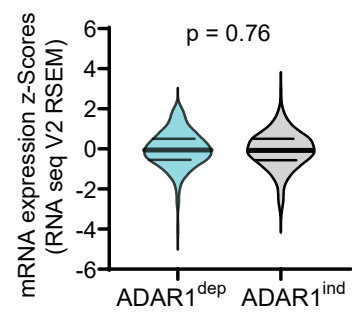

DDX3X

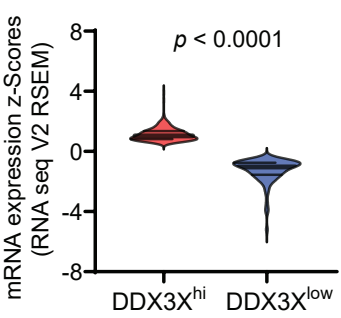

DDX3X

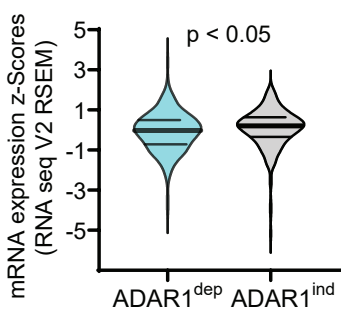

ADAR1

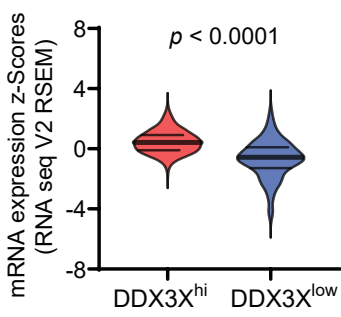

e response to virus se to type I interferon type I interferon signaling pathway response to type I interferon regulation of multi-organism process negative regulation of viral process regulation of innate immune response

negative regulation of viral life cycle positive regulation of cytokine production

response to virus defense response to virus

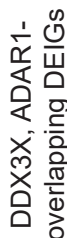
cellular response to type I interferon type I interferon signaling pathway response to type l interferon response to type I interferon
response to interferon-gamma negative regulation of viral life cycle negative regulation of viral life cycle
-gamma-mediated signaling pathway cellular response to interferon-gamma regulation of viral life cycle

positive regulation of cytokine production regulation of innate immune response $\mathrm{T}$ cell activation leukocyte proliferation regulation of immune effector process positive regulation of innate immune response regulation of inflammatory response lymphocyte differentiation lymphocyte differentiation
humoral immune response lymphocyte proliferation

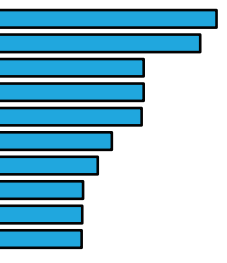

Differentially expressed immune-related genes (DEIGs)

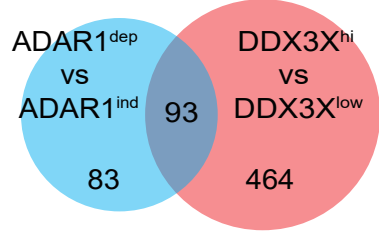

ADAR1 DEIGs

$\square$ DDX3X-ADAR1 DEIGs

$\square$ DDX3X DEIGs 
a

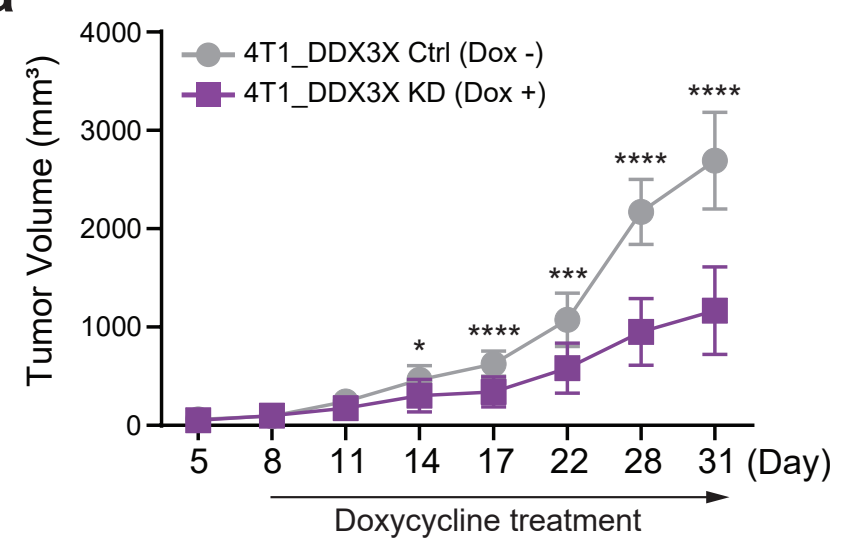

Fig. 7

bioRxiv preprint doi: https://doi.org/10.1101/2020.09.09.289587; this version posted September 10, 2020. The copyright holder for this preprint (which was not certified by peer review) is the aythor/funder. All rights reserved. No reuse allowed without peraission.
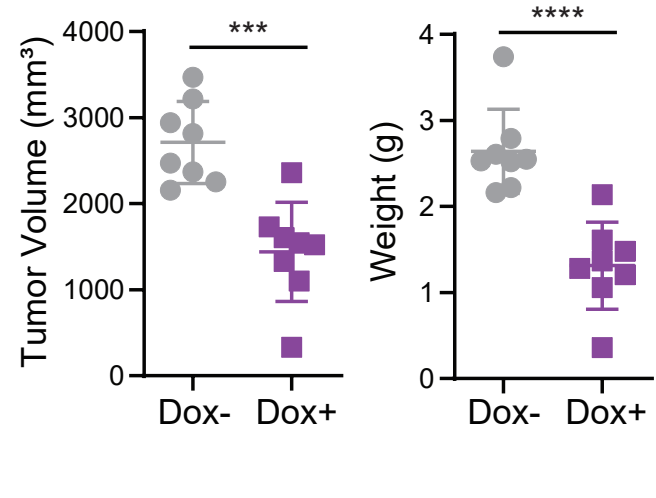

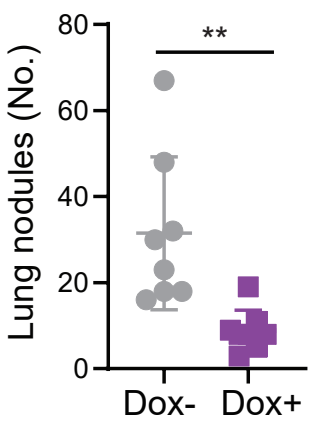

d

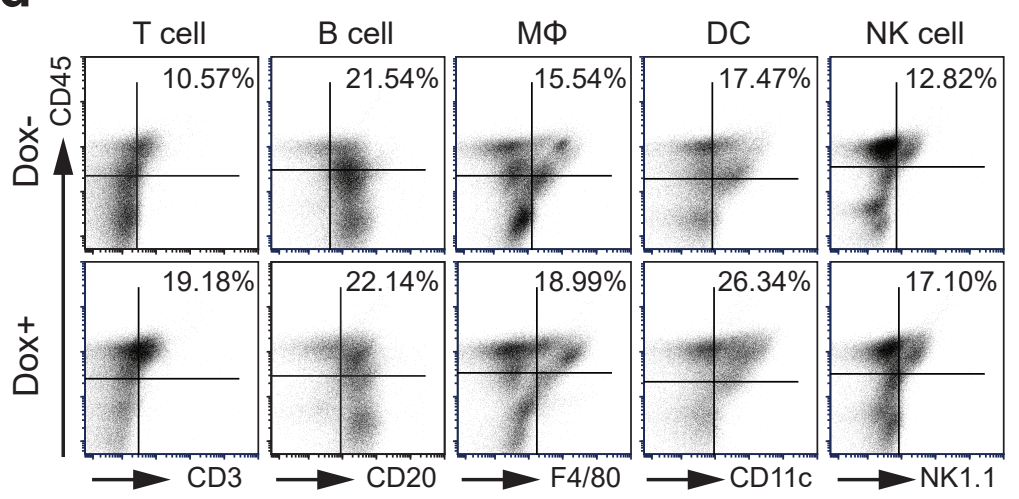

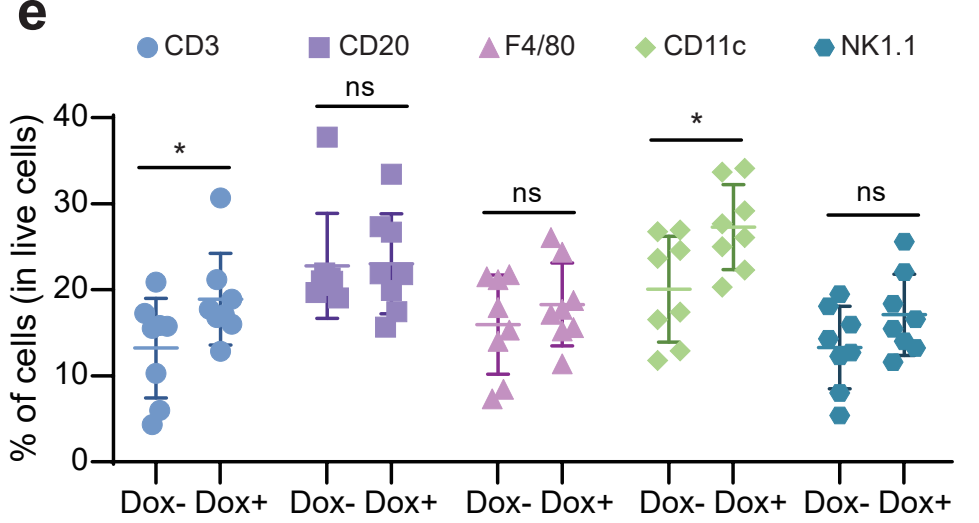

f

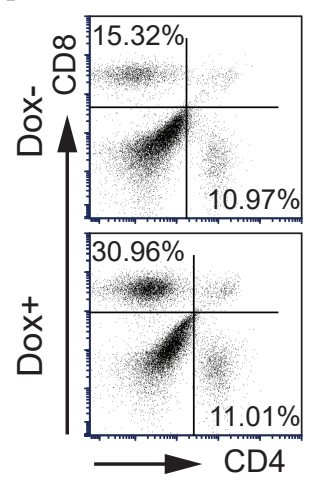

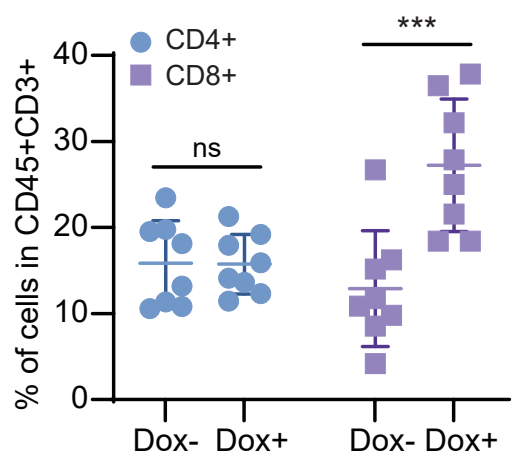

g

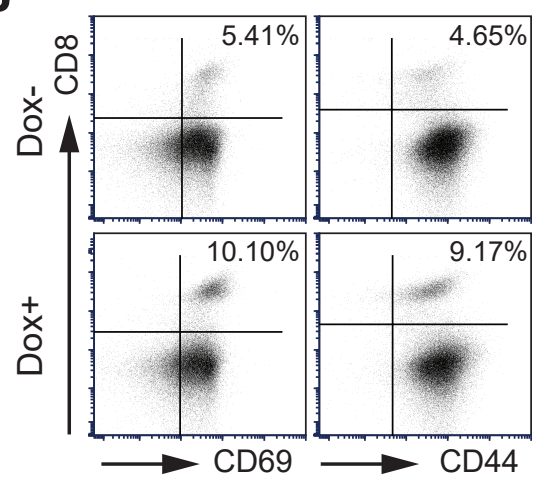

- CD3+CD8+CD69+

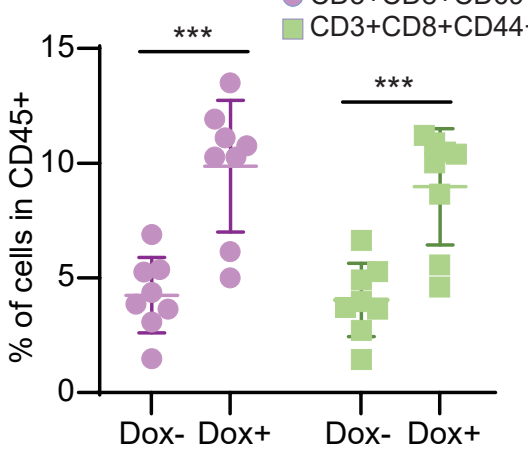

h

Ki-67

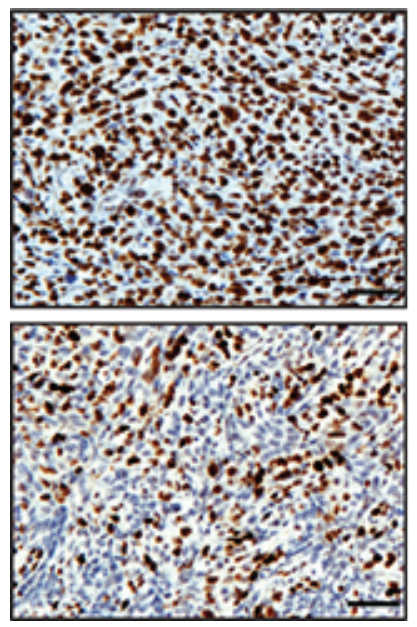

CD8

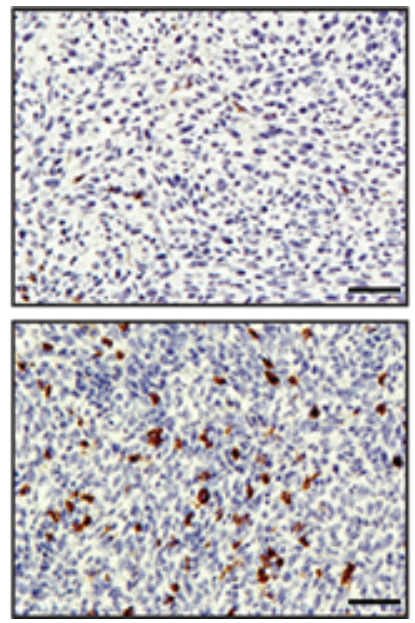

Perforin

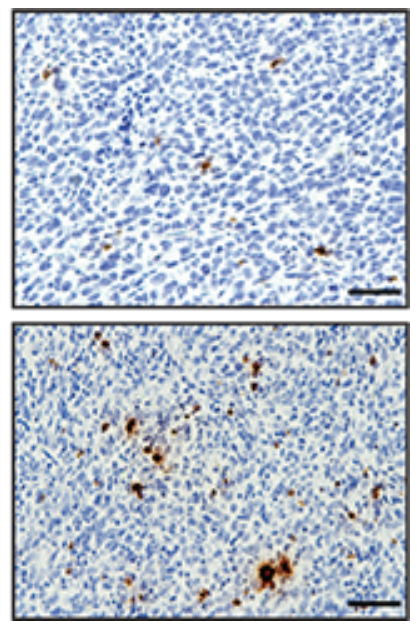

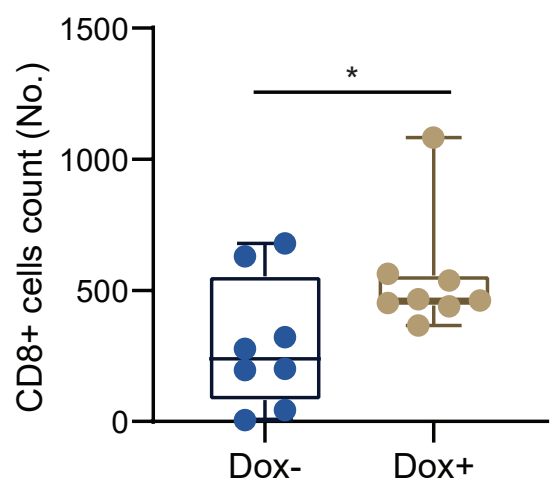

